\title{
Power corrections to symmetric point vertices in Gribov-Zwanziger theory
}

\author{
J.A. Gracey, \\ Theoretical Physics Division, \\ Department of Mathematical Sciences, \\ University of Liverpool, \\ P.O. Box 147, \\ Liverpool, \\ L69 3BX, \\ United Kingdom.
}

\begin{abstract}
The 3-point vertices of QCD are examined at the symmetric subtraction point at one loop in the Landau gauge in the presence of the Gribov mass, $\gamma$. They are expanded in powers of $\gamma^{2}$ up to dimension four in order to determine the order of the leading correction. As well as analysing the pure Gribov-Zwanziger Lagrangian, its extensions to include localizing ghost masses are also examined. For comparison a pure gluon mass term is also considered.
\end{abstract}




\section{Introduction.}

In the late 1990's an interesting property of the running of an effective coupling constant derived from the triple gluon vertex in Quantum Chromodynamics (QCD) was revealed. In several articles, [1, 2, 3, 4, 5, using lattice gauge theory techniques and restricting to the Landau gauge the effective coupling constant appeared to deviate from the expected behaviour in an energy range intermediate between high and low. Moreover the deviation between expected and measured behaviour could be fitted by a power law correction. While such discrepancies are not ordinarily unexpected in essence because of our knowledge of the operator product expansion, the power correction was claimed to correspond to a dimension two operator rather than a dimension four one, [1, 2, 3, 4, 5]. The latter is usually associated with the vacuum expectation value of the square of the gluon field strength, which is gauge invariant, and is termed the gluon condensate. However, it was proposed that the operator associated with the dimension two correction was $\frac{1}{2} A_{\mu}^{a 2}$ where $A_{\mu}^{a}$ is the gauge potential. Such a gauge variant operator is not excluded as ultimately the running coupling constant is not a physical quantity. Subsequent to this there has been interest in trying to understand this dimension two operator both in the coupling constant situation and other quantities such as the gluon and Faddeev-Popov ghost propagators in the infrared. The condensation of a dimension two operator could be related to confinement in the sense that it generates an effective gluon mass. See, for example, [6] for a summary. Thus in the infrared the gluon propagator will freeze to a finite non-zero value at zero momentum. This is in accord with a long-standing result of Cornwall, [7], who established that the frozen gluon propagator follows from the dynamical generation of an effective mass which is momentum dependent. In more recent years progress with Landau gauge lattice computations has produced data which actually appears to support a frozen gluon propagator. For instance, see the early activity in this respect [8, 9, 10, 11, 12, 13, 14, 15, 16]. This should be qualified by the remark that ensuring that one is in a properly fixed gauge free from the complications of Gribov copies, [17], is a non-trivial exercise. Indeed it is perhaps fair to comment that this has yet to be fully resolved. Also the zero momentum regime is numerically difficult to achieve. Aside from the gluon propagator the Faddeev-Popov ghost propagator has also been studied in the Landau gauge. It too can be used to study an effective running coupling constant at zero momentum via the ghost-gluon vertex which exploits properties derived from the Slavnov-Taylor identities, [18].

From the analytic point of view one can focus on the Landau gauge in the infrared. The seminal work in this area was by Gribov, [17, who highlighted the inability to fix the gauge globally due to the presence of Gribov copies. One property he established was that a less incorrectly fixed Landau gauge could be effected with a modification of the Yang-Mills action. This restricted the path integral to the first Gribov region and introduced a non-local operator into the Lagrangian. Although a semi-classical approach was used in [17 the non-locality was localized in a series of articles by Zwanziger, [19, 20, 21, 22, 23, 24, 25, 26, 27, to produce a local renormalizable Lagrangian. This construction meant that one could compute in the Gribov context and study infrared behaviour. Already noted in [17] the copies introduced a new mass called the Gribov mass, $\gamma$, which is not an independent parameter as it satisfies a gap equation. Its presence ensures that the Faddeev-Popov ghost propagator enhances and the gluon propagator is suppressed in that it vanishes at zero momentum. While this does not accord with recent lattice data, modifications of the original Gribov-Zwanziger Lagrangian, 28, 29, 30, have been developed which do model the frozen gluon and non-enhanced Faddeev-Popov ghost propagator. Though such refinements are not unique. In [30] it was demonstrated that several different localizing ghost condensations can model a frozen gluon. However each has a different prediction for other quantities which are not yet or as widely measured on the lattice. While 
there has been an intense amount of lattice activity on the propagators with as yet no resolution as to which refined solution is the leading candidate, the presence of an independent mass scale in the pure or refined Gribov-Zwanziger Lagrangians could be the source of the power corrections of the effective coupling constants of [1, 2, 3, 4, 5]. Therefore, it is the purpose of this article to explore this possibility and produce the one loop corrections to 3-point vertices of QCD using the Gribov-Zwanziger setup. We will do this for the specific momentum configuration of having the three external legs at non-zero momenta and with squared momenta all equal. This is known as the symmetric point and is a non-exceptional momentum configuration. Hence it will be free of infrared ambiguities which could plague the asymmetric subtraction point calculation which is an exceptional configuration.

One advantage of this symmetric subtraction point is that by considering non-zero external momenta one will avoid having to go to the far infrared which is beset with potential gauge fixing issues. In essence the momentum range we have in mind for comparisons to numerical work is the intermediate one where the explorations of [1] were centred. In other words one is in a next to high energy approximation where one can formally access power corrections. At much lower energies the explicit expressions for vertex amplitudes would be complicated functions of the masses and momenta. These would then have to be expanded in a power series to reveal power corrections. This intermediate energy range is important from the Feynman diagram point of view. The main reason for this is that we can expand the Feynman graphs in a power series in a mass scale. This avoids having to actually determine the explicit complicated functions and then expand them. Central to our calculations will be the use of the method of [31] which allows us to correctly power expand Feynman integrals. Given that the Gribov mass is the associated scale in the pure Gribov-Zwanziger case there is no a priori reason to exclude it as being a potential source for the power corrections advanced in [1, 2, 3, 4, 5. For instance, one could in principle relate $\gamma^{2}$ to the vacuum expectation value of $\frac{1}{2} A_{\mu}^{a 2}$ precisely because $\gamma^{2}$ appears in the gluon propagator, [17]. Hence $\left\langle\frac{1}{2} A_{\mu}^{a}{ }^{2}\right\rangle$ will be proportional to $\gamma^{2}$ on dimensional grounds but both will be as indistinguishable from the other as the dimension two correction measured on the lattice. Thus in our exploration of this problem the aim will not only be the determination of whether there are dimension two, four or higher corrections but also to deduce the magnitude and sign of the coefficient. In studying the triple gluon, ghost-gluon and quark-gluon vertices we will be able to devise a test which in principle could distinguish which of the refined solutions if any is favourable but crucially in a regime where deep infrared issues, such as Gribov copies, do not complicate a lattice study. However, as a control on the Gribov mass investigation we will also repeat the same analysis for the case where the gluon has an explicit mass, $m$. Ordinarily such a naive mass term breaks gauge invariance but one can have a non-local but gauge invariant gluon mass term. (See, for instance, [32, 33].) This operator reduces to $\frac{1}{2} m^{2} A_{\mu}^{a}$ in the Landau gauge. Having such a control calculation to compare with the Gribov mass case is important as a gluon mass also mimicks a frozen gluon propagator. Thus that could actually be the source of the lattice computations. If that were the case then a symmetric vertex study could be used as a confirmatory test.

The paper is organized as follows. In section 2 we discuss the general background to the Gribov-Zwanziger Lagrangian and its extensions which are needed for the computations we carry out. This includes the formalism we use to construct the three vertex functions at the symmetric point. Section 3 focuses on general aspects of the calculation including how the Feynman integrals are deduced in a power series expansion using the technique devised in [31]. The subsequent four sections are devoted to recording the results for the pure Gribov-Zwanziger case, what is termed the $\mathcal{Q}$ and $\mathcal{R}$ solutions and a pure gluon mass respectively. We discuss our test in the concluding section. Several appendices provide technical details which supplement the main discussion. 


\section{Background.}

We begin by recalling the main aspects of the Gribov-Zwanziger construction which are necessary for our computations. In [17] the Yang-Mills action was modified in order to take into account the ambiguities which arise in the gauge fixing procedure. With the restriction of the path integral to the first Gribov region the modification defines a boundary termed the Gribov horizon which manifests itself as a non-local dimension zero operator in the Lagrangian. It is related to the Faddeev-Popov operator, $\left(\partial^{\mu} D_{\mu}\right)^{a b}$, in that the boundary corresponds to the surface defined by the first zeros of the operator. Thus the inverse operator will be infinite at the Gribov horizon and hence as long as there are no poles in the inverse one is within the Gribov region, [17. Originally in [17, the action was treated in a semi-classical approximation in such a way that only the leading term of the Faddeev-Popov operator was used to define the boundary in the path integral. Subsequently, Zwanziger extended the analysis to all orders to produce the inverse Faddeev-Popov operator, [21]. The resulting Lagrangian is, [26],

$$
L^{\text {Gribov }}=L^{\mathrm{QCD}}+\frac{\gamma^{4}}{2} f^{e a c} f^{e b d} A_{\mu}^{a}(x)\left(\frac{1}{\partial^{\nu} D_{\nu}}\right)^{c d} A^{b \mu}(x)-\frac{d N_{A} \gamma^{4}}{2 g^{2}}
$$

where $g$ is the coupling constant, $d$ is the spacetime dimension, $N_{A}$ is the dimension of the adjoint representation of the colour group whose structure constants are $f^{a b c}$. Here

$$
L^{\mathrm{QCD}}=-\frac{1}{4} G_{\mu \nu}^{a} G^{a \mu \nu}-\frac{1}{2 \alpha}\left(\partial^{\mu} A_{\mu}^{a}\right)^{2}-\bar{c}^{a} \partial^{\mu} D_{\mu} c^{a}+i \bar{\psi}^{i I} \not D \psi^{i I}
$$

is the usual QCD Lagrangian valid at high energy where $c^{a}$ is the Faddeev-Popov ghost and $\psi^{i I}$ are massless quarks. Although we have included the gauge parameter $\alpha$ associated with the linear covariant gauge fixing we will perform all our calculations in the Landau gauge which is $\alpha=0$. In (2.1) the mass parameter $\gamma$ is known as the Gribov mass and is not an independent parameter as it satifies a gap equation derived from the horizon, (2.3). Only when $\gamma$ satisfies the gap equation is one actually in the gauge theory [17, 21, 26]. The field independent term of (2.1) ensures the non-triviality of the condition since, [26],

$$
f^{e a c} f^{e b d}\left\langle A_{\mu}^{a}(x)\left(\frac{1}{\partial^{\nu} D_{\nu}}\right)^{c d} A^{b \mu}(x)\right\rangle=\frac{d N_{A}}{g^{2}} .
$$

The presence of the non-local operator implies that the gluon propagator is modified from that which is used at high energy. Specifically, [17,

$$
\left\langle A_{\mu}^{a}(p) A_{\nu}^{b}(-p)\right\rangle=-\frac{\delta^{a b} p^{2}}{\left[\left(p^{2}\right)^{2}+C_{A} \gamma^{4}\right]} P_{\mu \nu}(p)
$$

which behaves as $-\frac{P_{\mu \nu}(p)}{p^{2}} \delta^{a b}$ at large momenta where

$$
P_{\mu \nu}(p)=\eta_{\mu \nu}-\frac{p_{\mu} p_{\nu}}{p^{2}}
$$

At low momenta the propagator tends to zero which is the gluon suppression, [17].

While (2.1) extends the Yang-Mills action to incorporate the copy issue, from a practical point of view the non-local term means that one cannot use it for explicit calculations. To circumvent this Zwanziger managed to localize the non-locality in several articles, 20, 21, 26], to produce a local Lagrangian. Its renormalizability was established in [26, 34, 35]. In order to 
achieve the localization localizing ghost fields were introduced, [26], so that (2.1) is replaced by

$$
\begin{aligned}
L^{\mathrm{GZ}}= & L^{\mathrm{QCD}}+\frac{1}{2} \rho^{a b \mu} \partial^{\nu}\left(D_{\nu} \rho_{\mu}\right)^{a b}+\frac{i}{2} \rho^{a b \mu} \partial^{\nu}\left(D_{\nu} \xi_{\mu}\right)^{a b}-\frac{i}{2} \xi^{a b \mu} \partial^{\nu}\left(D_{\nu} \rho_{\mu}\right)^{a b} \\
& +\frac{1}{2} \xi^{a b \mu} \partial^{\nu}\left(D_{\nu} \xi_{\mu}\right)^{a b}-\bar{\omega}^{a b \mu} \partial^{\nu}\left(D_{\nu} \omega_{\mu}\right)^{a b}-\frac{1}{\sqrt{2}} g f^{a b c} \partial^{\nu} \bar{\omega}_{\mu}^{a e}\left(D_{\nu} c\right)^{b} \rho^{e c \mu} \\
& -\frac{i}{\sqrt{2}} g f^{a b c} \partial^{\nu} \bar{\omega}_{\mu}^{a e}\left(D_{\nu} c\right)^{b} \xi^{e c \mu}-i \gamma^{2} f^{a b c} A^{a \mu} \xi_{\mu}^{b c}-\frac{d N_{A} \gamma^{4}}{2 g^{2}} .
\end{aligned}
$$

Here $\rho_{\mu}^{a b}$ and $\xi_{\mu}^{a b}$ are real fields and we have chosen this version over the complex fields of the earlier localization, [20, 21, 26]. Accompanying these bosonic fields are the other localizing ghosts, $\omega_{\mu}^{a b}$ and $\bar{\omega}_{\mu}^{a b}$, which are Grassmann. They are required to ensure that the ultraviolet structure of the theory such as asymptotic freedom is not upset nor renormalizability lost by the sole presence of the bosonic ghosts. The other main feature is that the dimension zero operator of (2.1) is translated into a dimension two mass-like term which mixes $A_{\mu}^{a}$ and $\xi_{\mu}^{a b}$. In effect this term corresponds to the non-local term which can be clearly seen by recalling the relation

$$
A_{\mu}^{a}=-\frac{i}{C_{A} \gamma^{2}} f^{a b c}\left(\partial^{\nu} D_{\nu} \xi_{\mu}\right)^{b c}
$$

which is deduced from the $\xi_{\mu}^{a b}$ equation of motion. The original horizon condition (2.3) becomes

$$
f^{a b c}\left\langle A^{a \mu}(x) \xi_{\mu}^{b c}(x)\right\rangle=\frac{i d N_{A} \gamma^{2}}{g^{2}}
$$

in (2.6). With the extra fields there is an extended set of propagators which are, [26],

$$
\begin{aligned}
\left\langle A_{\mu}^{a}(p) A_{\nu}^{b}(-p)\right\rangle & =-\frac{\delta^{a b} p^{2}}{\left[\left(p^{2}\right)^{2}+C_{A} \gamma^{4}\right]} P_{\mu \nu}(p),\left\langle A_{\mu}^{a}(p) \xi_{\nu}^{b c}(-p)\right\rangle=\frac{i f^{a b c} \gamma^{2}}{\left[\left(p^{2}\right)^{2}+C_{A} \gamma^{4}\right]} P_{\mu \nu}(p) \\
\left\langle\xi_{\mu}^{a b}(p) \xi_{\nu}^{c d}(-p)\right\rangle & =-\frac{\delta^{a c} \delta^{b d}}{p^{2}} \eta_{\mu \nu}+\frac{f^{a b e} f^{c d e} \gamma^{4}}{p^{2}\left[\left(p^{2}\right)^{2}+C_{A} \gamma^{4}\right]} P_{\mu \nu}(p),\left\langle A_{\mu}^{a}(p) \rho_{\nu}^{b c}(-p)\right\rangle=0 \\
\left\langle\rho_{\mu}^{a b}(p) \rho_{\nu}^{c d}(-p)\right\rangle & =\left\langle\omega_{\mu}^{a b}(p) \bar{\omega}_{\nu}^{c d}(-p)\right\rangle=-\frac{\delta^{a c} \delta^{b d}}{p^{2}} \eta_{\mu \nu},\left\langle\xi_{\mu}^{a b}(p) \rho_{\nu}^{c d}(-p)\right\rangle=0 .
\end{aligned}
$$

The suppressed gluon propagator emerges again. Despite the presence of the mixed propagator it is possible to compute one and two loop corrections to various quantities such as the gap equation for $\gamma$, [17, 26, 36].

While this was the standard Lagrangian used to incorporate Gribov copies it did not cover the observed behaviour on the lattice since the gluon propagator does not freeze to zero but to a finite non-zero value. To account for this in the Gribov-Zwanziger context various extensions to (2.6) have been considered in [28, 29, 30]. Each generalization can be summarized in the addition of a mass operator for the localizing ghost sector. The most general such operator is, [30,

$$
\mathcal{O}=\left[\mu_{\mathcal{Q}}^{2} \delta^{a c} \delta^{b d}+\mu_{\mathcal{W}}^{2} f^{a c e} f^{b d e}+\frac{\mu_{\mathcal{R}}^{2}}{C_{A}} f^{a b e} f^{c d e}+\mu_{\mathcal{S}}^{2} d_{A}^{a b c d}+\frac{\mu_{\mathcal{P}}^{2}}{N_{A}} \delta^{a b} \delta^{c d}+\mu_{\mathcal{T}}^{2} \delta^{a d} \delta^{b c}\right] \mathcal{O}^{a b c d}
$$

where

$$
\mathcal{O}^{a b c d}=\frac{1}{2}\left[\rho^{a b} \rho^{c d}+i \xi^{a b} \rho^{c d}-i \rho^{a b} \xi^{c d}+\xi^{a b} \xi^{c d}\right]-\bar{\omega}^{a b} \omega^{c d} .
$$

Each colour structure is tagged with a mass, $\mu_{\mathcal{I}}$, where we use the same labels and conventions as were used in [30]. The tensor $d_{A}^{a b c d}$ is totally symmetric and is defined by, [37],

$$
d_{A}^{a b c d}=\frac{1}{6} \operatorname{Tr}\left(T_{A}^{a} T_{A}^{(b} T_{A}^{c} T_{A}^{d)}\right) .
$$


Overall the additional operator is BRST invariant and satisfies a Slavnov-Taylor identity so that the operator renormalization constant is related to the $\xi_{\mu}^{a b}$ wave function renormalization constant, 28, 29, 30]. With this additional term the propagators (2.9) become significantly more complicated. These were analysed at length in [30] where the full set for $S U(3)$ were recorded explicitly. In general and for certain specific cases the extra mass parameters $\mu_{\mathcal{I}}$ lead to gluon propagators which freeze to a non-zero finite value. In [29] one of these cases was examined which in the notation of (2.10) was termed the $\mathcal{Q}$ solution. However, in 30] it was noted that this solution was not unique and that another specific single mass parameter solution could be equally viable. This was the $\mathcal{R}$ case. It was argued in [30] that this was a more natural solution than the $\mathcal{Q}$ case since a non-zero $\mu_{\mathcal{R}}$ would correspond to a condensation of the operator $\xi_{\mu}^{a b} \xi^{a b \mu}$ consistent with the structure of the pure Gribov-Zwanziger propagators, [30]. To see the frozen gluon propagator at the outset the propagators for these two specific solutions are

$$
\begin{aligned}
\left\langle A_{\mu}^{a}(p) A_{\nu}^{b}(-p)\right\rangle_{\mathcal{Q}} & =-\frac{\delta^{a b}\left[p^{2}+\mu_{\mathcal{Q}}^{2}\right]}{\left[\left(p^{2}\right)^{2}+\mu_{\mathcal{Q}}^{2} p^{2}+C_{A} \gamma^{4}\right]} P_{\mu \nu}(p),\left\langle A_{\mu}^{a}(p) \rho_{\nu}^{b c}(-p)\right\rangle_{\mathcal{Q}}=0 \\
\left\langle A_{\mu}^{a}(p) \xi_{\nu}^{b c}(-p)\right\rangle_{\mathcal{Q}} & =\frac{i f^{a b c} \gamma^{2}}{\left[\left(p^{2}\right)^{2}+\mu_{\mathcal{Q}}^{2} p^{2}+C_{A} \gamma^{4}\right]} P_{\mu \nu}(p),\left\langle\xi_{\mu}^{a b}(p) \rho_{\nu}^{c d}(-p)\right\rangle_{\mathcal{Q}}=0 \\
\left\langle\xi_{\mu}^{a b}(p) \xi_{\nu}^{c d}(-p)\right\rangle_{\mathcal{Q}} & =-\frac{\delta^{a c} \delta^{b d}}{\left[p^{2}+\mu_{\mathcal{Q}}^{2}\right]} \eta_{\mu \nu}+\frac{f^{a b e} f^{c d e} \gamma^{4}}{\left[p^{2}+\mu_{\mathcal{Q}}^{2}\right]\left[\left(p^{2}\right)^{2}+\mu_{\mathcal{Q}}^{2} p^{2}+C_{A} \gamma^{4}\right]} P_{\mu \nu}(p) \\
\left\langle\rho_{\mu}^{a b}(p) \rho_{\nu}^{c d}(-p)\right\rangle_{\mathcal{Q}} & =\left\langle\omega_{\mu}^{a b}(p) \bar{\omega}_{\nu}^{c d}(-p)\right\rangle_{\mathcal{Q}}=-\frac{\delta^{a c} \delta^{b d}}{\left[p^{2}+\mu_{\mathcal{Q}}^{2}\right]} \eta_{\mu \nu}
\end{aligned}
$$

and

$$
\begin{aligned}
\left\langle A_{\mu}^{a}(p) A_{\nu}^{b}(-p)\right\rangle_{\mathcal{R}}= & -\frac{\delta^{a b}\left[p^{2}+\mu_{\mathcal{R}}^{2}\right]}{\left[\left(p^{2}\right)^{2}+\mu_{\mathcal{R}}^{2} p^{2}+C_{A} \gamma^{4}\right]} P_{\mu \nu}(p),\left\langle A_{\mu}^{a}(p) \rho_{\nu}^{b c}(-p)\right\rangle_{\mathcal{R}}=0 \\
\left\langle A_{\mu}^{a}(p) \xi_{\nu}^{b c}(-p)\right\rangle_{\mathcal{R}}= & \frac{i f^{a b c} \gamma^{2}}{\left[\left(p^{2}\right)^{2}+\mu_{\mathcal{R}}^{2} p^{2}+C_{A} \gamma^{4}\right]} P_{\mu \nu}(p),\left\langle\xi_{\mu}^{a b}(p) \rho_{\nu}^{c d}(-p)\right\rangle_{\mathcal{R}}=0 \\
\left\langle\xi_{\mu}^{a b}(p) \xi_{\nu}^{c d}(-p)\right\rangle_{\mathcal{R}}= & -\frac{\delta^{a c} \delta^{b d}}{p^{2}} \eta_{\mu \nu}+\frac{f^{a b e} f^{c d e}\left[\mu_{\mathcal{R}}^{2} p^{2}+C_{A} \gamma^{4}\right]}{C_{A} p^{2}\left[\left(p^{2}\right)^{2}+\mu_{\mathcal{R}}^{2} p^{2}+C_{A} \gamma^{4}\right]} P_{\mu \nu}(p) \\
& +\frac{f^{a b e} f^{c d e} \mu_{\mathcal{R}}^{2}}{C_{A} p^{2}\left[p^{2}+\mu_{\mathcal{R}}^{2}\right]} L_{\mu \nu}(p) \\
\left\langle\rho_{\mu}^{a b}(p) \rho_{\nu}^{c d}(-p)\right\rangle_{\mathcal{R}}= & \left\langle\omega_{\mu}^{a b}(p) \bar{\omega}_{\nu}^{c d}(-p)\right\rangle_{\mathcal{R}}=-\frac{\delta^{a c} \delta^{b d}}{p^{2}} \eta_{\mu \nu}+\frac{f^{a b e} f^{c d e} \mu_{\mathcal{R}}^{2}}{C_{A} p^{2}\left[p^{2}+\mu_{\mathcal{R}}^{2}\right]} \eta_{\mu \nu} .
\end{aligned}
$$

It is these propagators which we will use as part of our study of the power corrections to the 3 -point vertices. While the gluon sectors of each are formally equivalent the key differences are in the localizing ghost propagators. In the $\mathcal{Q}$ case there are no massless factors in any of the propagators whereas there are massless modes in the $\mathcal{R}$ solution. In [30] it was noted that this led to different infrared properties of the localizing ghost propagators after the gap equation for $\gamma$ is satisfied. Such differing behaviour can be used to distinguish from these solutions if lattice data was available for the localizing ghost propagators.

We now turn to the formalism relating to specific Green's functions we will compute in a power series expansion. These are

$$
\begin{aligned}
\left.\left\langle A_{\mu}^{a}(p) A_{\nu}^{b}(q) A_{\sigma}^{c}(r)\right\rangle\right|_{p^{2}=q^{2}=-\mu^{2}} & =\left.f^{a b c} \Sigma_{\mu \nu \sigma}^{\mathrm{ggg}}\left(p, q, \gamma^{2}, \mu_{\mathcal{I}}^{2}\right)\right|_{p^{2}=q^{2}=-\mu^{2}} \\
\left.\left\langle c^{a}(p) \bar{c}^{b}(q) A_{\sigma}^{c}(r)\right\rangle\right|_{p^{2}=q^{2}=-\mu^{2}} & =\left.f^{a b c} \Sigma_{\sigma}^{\mathrm{ccg}}\left(p, q, \gamma^{2}, \mu_{\mathcal{I}}^{2}\right)\right|_{p^{2}=q^{2}=-\mu^{2}} \\
\left.\left\langle\psi^{i}(p) \bar{\psi}^{j}(q) A_{\sigma}^{c}(r)\right\rangle\right|_{p^{2}=q^{2}=-\mu^{2}} & =\left.T_{i j}^{c} \Sigma_{\sigma}^{\mathrm{qqg}}\left(p, q, \gamma^{2}, \mu_{\mathcal{I}}^{2}\right)\right|_{p^{2}=q^{2}=-\mu^{2}}
\end{aligned}
$$


where

$$
r=-p-q
$$

and each Green's function corresponds respectively to the triple gluon, ghost-gluon and quarkgluon vertices. The independent external momenta flowing in through two of the external legs are $p$ and $q$ and neither are nullified so that we are in a non-exceptional momentum configuration which does not suffer from infrared issues. All squared momenta are held at the same value

$$
p^{2}=q^{2}=r^{2}=-\mu^{2}
$$

which implies

$$
p q=\frac{1}{2} \mu^{2}
$$

where $\mu$ is the mass scale introduced to ensure that the coupling constant remains dimensionless in $d$-dimensional spacetime as we will be using dimensional regularization throughout. Our regularizing parameter will be $\epsilon$ where $d=4-2 \epsilon$. As each Green's function carries colour and Lorentz indices we have to decompose them into scalar amplitudes. For the former we have done this in (2.15). This is relatively straightforward since to the loop order we are working there is only one colour tensor for each vertex function which is evident from the explicit computations. For the Lorentz sector we have to introduce a set of basis tensors which are built from $\eta_{\mu \nu}$ and $p_{\mu}$ and $q_{\mu}$. Thus

$$
\begin{aligned}
\left.\Sigma_{\mu \nu \sigma}^{\mathrm{ggg}}\left(p, q, \gamma^{2}, \mu_{\mathcal{I}}^{2}\right)\right|_{p^{2}=q^{2}=-\mu^{2}} & =\sum_{k=1}^{14} \mathcal{P}_{(k) \mu \nu \sigma}^{\mathrm{ggg}}(p, q) \Sigma_{(k)}^{\mathrm{ggg}}\left(p, q, \gamma^{2}, \mu_{\mathcal{I}}^{2}\right) \\
\left.\Sigma_{\sigma}^{\mathrm{ccg}}\left(p, q, \gamma^{2}, \mu_{\mathcal{I}}^{2}\right)\right|_{p^{2}=q^{2}=-\mu^{2}} & =\sum_{k=1}^{2} \mathcal{P}_{(k) \sigma}^{\mathrm{ccg}}(p, q) \Sigma_{(k)}^{\mathrm{ccg}}\left(p, q, \gamma^{2}, \mu_{\mathcal{I}}^{2}\right) \\
\left.\sum_{\sigma}^{\mathrm{qqg}}\left(p, q, \gamma^{2}, \mu_{\mathcal{I}}^{2}\right)\right|_{p^{2}=q^{2}=-\mu^{2}} & =\sum_{k=1}^{6} \mathcal{P}_{(k) \sigma}^{\mathrm{qqg}}(p, q) \Sigma_{(k)}^{\mathrm{qqg}}\left(p, q, \gamma^{2}, \mu_{\mathcal{I}}^{2}\right)
\end{aligned}
$$

which defines the scalar amplitudes for each vertex function. The explicit tensors for each case are given in Appendix A and we use the same set as was used in [38. We note that away from the symmetric point restriction the basis will involve more tensors. Also the basis is not unique and there are other choices. It turns out that for the triple gluon vertex from the explicit computations of [38] we have checked that to two loops one can write the vertex function more compactly in terms of three tensors. One of these, for instance, corresponds to the Feynman rule of the vertex itself when $r=-p-q$. The other two do not involve $\eta_{\mu \nu}$ and their explicit forms are given in Appendix A. Therefore, here we choose to work in this more compact basis and replace the first equation of (2.15) by

$$
\left.\tilde{\Sigma}_{\mu \nu \sigma}^{\mathrm{ggg}}\left(p, q, \gamma^{2}, \mu_{\mathcal{I}}^{2}\right)\right|_{p^{2}=q^{2}=-\mu^{2}}=\sum_{k=1}^{3} \tilde{\mathcal{P}}_{(k) \mu \nu \sigma}^{\mathrm{ggg}}(p, q) \tilde{\Sigma}_{(k)}^{\mathrm{ggg}}\left(p, q, \gamma^{2}, \mu_{\mathcal{I}}^{2}\right) .
$$

To distinguish this basis from the previous set the amplitudes will have a tilde. In order to calculate each scalar amplitude we use the same projection approach as [38. Briefly each Green's function is multiplied by a linear combination of the basis tensors in $d$-dimensions. The coefficients are found by first constructing the matrix of products of all the basis tensors. Then the inverse of this matrix gives the linear combination. Denoting this inverse matrix by $\mathcal{M}$ we have

$$
\begin{aligned}
f^{a b c} \tilde{\Sigma}_{(k)}^{\mathrm{ggg}}\left(p, q, \gamma^{2}, \mu_{\mathcal{I}}^{2}\right) & =\left.\tilde{\mathcal{M}}_{k l}^{\mathrm{ggg}}\left(\tilde{\mathcal{P}}_{(l)}^{\mathrm{ggg} \mu \nu \sigma}(p, q)\left\langle A_{\mu}^{a}(p) A_{\nu}^{b}(q) A_{\sigma}^{c}(r)\right\rangle\right)\right|_{p^{2}=q^{2}=-\mu^{2}} \\
f^{a b c} \Sigma_{(k)}^{\mathrm{ccg}}\left(p, q, \gamma^{2}, \mu_{\mathcal{I}}^{2}\right) & =\left.\mathcal{M}_{k l}^{\mathrm{ccg}}\left(\mathcal{P}_{(l)}^{\mathrm{ccg} \sigma}(p, q)\left\langle c^{a}(p) \bar{c}^{b}(q) A_{\sigma}^{c}(r)\right\rangle\right)\right|_{p^{2}=q^{2}=-\mu^{2}} \\
T_{i j}^{c} \Sigma_{(k)}^{\mathrm{qqg}}\left(p, q, \gamma^{2}, \mu_{\mathcal{I}}^{2}\right) & =\left.\mathcal{M}_{k l}^{\mathrm{qqg}}\left(\mathcal{P}_{(l)}^{\mathrm{qqg} \sigma}(p, q)\left\langle\psi^{i}(p) \bar{\psi}^{j}(q) A_{\sigma}^{c}(r)\right\rangle\right)\right|_{p^{2}=q^{2}=-\mu^{2}}
\end{aligned}
$$


where $k$ and $l$ are the matrix labels. The explicit matrices are given in Appendix A. For the quark sector we have the additional issue of spinor indices to account for in the amplitude decomposition. So as well as building the tensor basis from $\eta_{\mu \nu}, p_{\mu}$ and $q_{\mu}$ one has also to include $\gamma_{\mu}$. This implies that products of $\gamma$-matrices can arise in the tensor basis and since we will be working in $d$-dimensions it is natural to use the generalized basis of $\gamma$-matrices which spans spinor space in $d$-dimensions, [39, 40, 41]. These are denoted by $\Gamma_{(n)}^{\mu_{1} \ldots \mu_{n}}$ where $n$ is a positive integer and defined by

$$
\Gamma_{(n)}^{\mu_{1} \ldots \mu_{n}}=\gamma^{\left[\mu_{1}\right.} \ldots \gamma^{\left.\mu_{n}\right]}
$$

where $1 / n$ ! is included in the antisymmetrization. The algebra of these has been studied at length in [41, 42, 43]. Though for constructing the matrix $\mathcal{M}_{k l}^{\mathrm{qqg}}$ a useful property is, [42, 44],

$$
\operatorname{tr}\left(\Gamma_{(m)}^{\mu_{1} \ldots \mu_{m}} \Gamma_{(n)}^{\nu_{1} \ldots \nu_{n}}\right) \propto \delta_{m n} I^{\mu_{1} \ldots \mu_{m} \nu_{1} \ldots \nu_{n}}
$$

which partitions the matrix where $I^{\mu_{1} \ldots \mu_{m} \nu_{1} \ldots \nu_{n}}$ is the unit matrix on the generalized spinor space.

\section{Calculational method.}

We now detail the overall method we have used to determine the power corrections to the one loop vertices at the symmetric point in each of the various Lagrangians we are interested in. We have followed a general approach which allows us to construct routines for all possible mass configurations. For instance, examining the propagators all possible one loop 3-point functions will have at most three different non-zero mass scales. However, since we are at a symmetric point and there are massless poles in the propagator sets, there are actually only seven distinct basic mass distribution configuration we have to consider. If we define

$$
I\left(m_{1}^{2}, m_{2}^{2}, m_{3}^{2}\right)=\left.\int \frac{d^{d} k}{(2 \pi)^{d}} \frac{1}{\left[k^{2}+m_{1}^{2}\right]\left[(k-p)^{2}+m_{2}^{2}\right]\left[(k+q)^{2}+m_{3}^{2}\right]}\right|_{p^{2}=q^{2}=-\mu^{2}}
$$

then the basic master integral structures from the point of view of mass distribution across the propagators are $I(0,0,0), I\left(m_{1}^{2}, 0,0\right), I\left(m_{1}^{2}, m_{1}^{2}, 0\right), I\left(m_{1}^{2}, m_{2}^{2}, 0\right), I\left(m_{1}^{2}, m_{1}^{2}, m_{1}^{2}\right), I\left(m_{1}^{2}, m_{1}^{2}, m_{2}^{2}\right)$ and $I\left(m_{1}^{2}, m_{2}^{2}, m_{3}^{2}\right)$. Here none of the $m_{i}$ are equal. We note that (3.1) is the basic structure since any of the Gribov or Stingl type propagators can always be written as the product of two canonical propagators and then partial fractioned. The fact that we are at a symmetric subtraction point means that $I\left(m_{1}^{2}, m_{2}^{2}, m_{3}^{2}\right)$ is totally symmetric in its arguments. We have not included powers of the propagators in the definition of $I\left(m_{1}^{2}, m_{2}^{2}, m_{3}^{2}\right)$ as we use the standard approach of breaking up all the contributing Feynman graphs into scalar integrals. These are determined after the general projection method. Then numerator scalar products are rewritten in terms of the propagator factors. For one loop 3-point functions at the symmetric point there are no irreducible numerators. To proceed further we have to write these integrals, which may have propagators to a negative power or positive power greater than unity, in terms of the basic master integrals for each of the seven mass distributions. For the massless case we did this in [38] at one and two loops. For the massive cases we extend that approach which used the Laporta algorithm [44. That method, 44], uses integration by parts to establish algebraic relations between all the integrals which arise. As there is an overredundancy in the relations, one can relate all integrals down to a basic set known as masters. The values for these are determined by other methods and hence the whole calculation is complete. In terms of tools we have used the REDUZE implementation, 45], which uses the GiNAC symbolic manipulation system, 46], and is written in $\mathrm{C}++$. The REDuze package creates a database for each topology and mass 
distribution and one extracts the relations which are needed for the specific computation. Aside from the massless one used in 38] we have constructed five other one loop databases. That for $I\left(m_{1}^{2}, m_{2}^{2}, m_{3}^{2}\right)$ is not needed as no integral of this mass distribution arises for propagator powers other than unity. Once the databases are determined the required integral relations are written in Form, 47], and included in the automatic computation routine. To generate the Feynman graphs for each of the three vertices we use QGRAF, [48. For the Gribov-Zwanziger case there are 30 one loop graphs and for the other two vertices there are 3 one loop graphs in both cases.

Having broken down the computation of the amplitudes of each vertex into the basic master integrals we have to substitute the explicit values for each. In general terms at this stage there are three basic configurations which correspond to having one, two or three propagators. By this we mean that in the latter two cases any two or three of the propagators present in (3.1). For the one and two propagator cases for different masses these integrals are known exactly and their power series expansion can then be substituted after carrying out a Taylor expansion. For $I\left(m_{1}^{2}, m_{2}^{2}, m_{3}^{2}\right)$ itself the explicit result is not known for any combination of non-zero $m_{i}$ except when all three masses are zero. Therefore, since we are only interested in the power series expansion we follow the method of [31]. This method allows one to expand Feynman integrals in powers of $m^{2} / \mu^{2}$ where $m$ is a generic mass scale deriving from a propagator in the original integral and $\mu$ is our common scale here for the squared momentum of the external legs. While 31 detailed at length the expansion of the two loop self-energy topology as an example the method is general and we use the general formalism that was provided there. If in general we denote by $J$ one of our master integrals $I\left(m_{1}^{2}, m_{2}^{2}, m_{3}^{2}\right)$ with at least one $m_{i} \neq 0$ then the asymptotic expansion is, 31,

$$
J_{\Gamma} \sim \sum_{\lambda} J_{\Gamma / \lambda} \circ \mathcal{T}_{\left\{m_{i}\right\} ;\left\{q_{i}\right\}} J_{\lambda}
$$

We use similar notation as [31] and note that $\Gamma$ is the original Feynman diagram, $\lambda$ are subgraphs which arise in the asymptotic expansion and $m_{i}$ formally represent the masses on each propagator of $\Gamma$. (In 31] $\gamma$ was used for the subgraphs but we use $\lambda$ here to avoid confusion with the Gribov mass which is a parameter in the actual expansion.) These are necessary to counteract the infrared infinities which arise if one naively expands the original integral in powers of $1 / \mu^{2}$. That is always the first term in the expansion and in that case the subgraph $\lambda$ is the unit graph. The other non-unit graphs in the sum are constructed from all possible routings of the external momenta around the graph. In the two loop example detailed in 31 there was only one such momenta unlike the two here. However, it is straightforward to see that there are three such graphs since with two momenta there are three ways to route the external momenta. In other words in each of the three graphs one of the three propagators of (3.1) will have no external momenta. For each of these three cases the subgraph $\lambda$ is expanded in powers of the masses $m_{i}$ and the momenta $q_{i}$ external to that subgraph itself. The terms of this Taylor expansion,

denoted by $\mathcal{T}_{\left\{m_{i}\right\} ;\left\{q_{i}\right\}} J_{\lambda}$, are then substituted in the reduced diagram $J_{\Gamma / \lambda}$ before performing the integration over the loop momentum, [31]. For the expansion the leading term will involve massless 3-point integrals which can be reduced to the one loop massless master of [49] using the REDUZE database we have already constructed. The remaining three terms reduce to one loop massive tadpoles which are readily evaluated. In Appendix $\mathrm{C}$ we have given various examples of the expansion of master integrals which were used within our calculations.

\section{Power corrections in the Gribov-Zwanziger Lagrangian.}

We now turn to the mundane task of recording the explicit results for each of the three vertices for various versions of the Gribov-Zwanziger Lagrangian, its extensions and the gluon mass 
case*. In this section we focus on the pure Gribov-Zwanziger Lagrangian, (2.6), where there are no mass terms deriving from localizing ghost field masses. Throughout this and subsequent sections we give the symmetric vertices to and including the term which corresponds to the dimension four correction. While we could in principle include higher order corrections, there does not appear to be any practical reason to do this at present since such terms would be difficult to extract numerically from the lattice. First, we record that the triple gluon vertex structure for $(2.6)$ is

$$
\begin{aligned}
\tilde{\Sigma}_{(1)}^{g g g}\left(p, q, \gamma^{2}, 0\right)= & \tilde{\Sigma}_{(1)}^{g g g}(p, q, 0,0) \\
& +\left[\left[\frac{13}{6}+\frac{7 \pi^{2}}{36}-\frac{7}{24} \psi^{\prime}\left(\frac{1}{3}\right)-\frac{1}{2} \ln \left[\frac{C_{A} \gamma^{4}}{\mu^{4}}\right]\right] \frac{C_{A}^{2} \gamma^{4}}{\mu^{4}}+O\left(\frac{\gamma^{6}}{\mu^{6}}\right)\right] a \\
& +O\left(a^{2}\right) \\
\tilde{\Sigma}_{(2)}^{g g g}\left(p, q, \gamma^{2}, 0\right)= & \tilde{\Sigma}_{(2)}^{g g g}(p, q, 0,0) \\
& +\left[\frac{3 \pi}{32} \frac{C_{A}^{3 / 2} \gamma^{2}}{\mu^{2}}+\left[\frac{289}{144}+\frac{787}{192} \ln \left[\frac{C_{A} \gamma^{4}}{\mu^{4}}\right]\right] \frac{C_{A}^{2} \gamma^{4}}{\mu^{4}}+O\left(\frac{\gamma^{6}}{\mu^{6}}\right)\right] a+O\left(a^{2}\right) \\
\tilde{\Sigma}_{(3)}^{g g g}\left(p, q, \gamma^{2}, 0\right)= & \tilde{\Sigma}_{(3)}^{g g g}(p, q, 0,0) \\
& +\left[\frac{3 \pi}{32} \frac{C_{A}^{3 / 2} \gamma^{2}}{\mu^{2}}+\left[\frac{25}{18} \psi^{\prime}\left(\frac{1}{3}\right)-\frac{25}{27} \pi^{2}-\frac{2033}{576}+\frac{799}{192} \ln \left[\frac{C_{A} \gamma^{4}}{\mu^{4}}\right]\right] \frac{C_{A}^{2} \gamma^{4}}{\mu^{4}}\right. \\
& \left.+O\left(\frac{\gamma^{6}}{\mu^{6}}\right)\right] a+O\left(a^{2}\right) .
\end{aligned}
$$

Interestingly there is no dimension two correction for channel 1 which is the channel corresponding to the original triple gluon vertex Feynman rule. The other amplitudes have a dimension two correction and moreover, that term is the same in both cases. The explicit values of the $\gamma^{2}=0$ amplitudes for this and the other cases are given in Appendix B. For the ghost-gluon and quark-gluon vertices we have

$$
\begin{aligned}
\Sigma_{(1)}^{c c g}\left(p, q, \gamma^{2}, 0\right)= & \Sigma_{(1)}^{c c g}(p, q, 0,0) \\
& -\left[\frac{9 \pi}{32} \frac{C_{A}^{3 / 2} \gamma^{2}}{\mu^{2}}+\left[\frac{377}{2304}+\frac{1}{144} \psi^{\prime}\left(\frac{1}{3}\right)-\frac{1}{216} \pi^{2}-\frac{1}{12} \ln \left[\frac{C_{A} \gamma^{4}}{\mu^{4}}\right]\right] \frac{C_{A}^{2} \gamma^{4}}{\mu^{4}}\right. \\
& \left.+O\left(\frac{\gamma^{6}}{\mu^{6}}\right)\right] a+O\left(a^{2}\right) \\
\Sigma_{(2)}^{c c g}\left(p, q, \gamma^{2}, 0\right)= & \Sigma_{(2)}^{c c g}(p, q, 0,0) \\
+ & {\left[\frac{3 \pi}{16} \frac{C_{A}^{3 / 2} \gamma^{2}}{\mu^{2}}+\left[\frac{1}{144} \psi^{\prime}\left(\frac{1}{3}\right)-\frac{1}{216} \pi^{2}-\frac{7}{2304}-\frac{1}{48} \ln \left[\frac{C_{A} \gamma^{4}}{\mu^{4}}\right]\right] \frac{C_{A}^{2} \gamma^{4}}{\mu^{4}}\right.} \\
& \left.+O\left(\frac{\gamma^{6}}{\mu^{6}}\right)\right] a+O\left(a^{2}\right)
\end{aligned}
$$

and

$$
\begin{aligned}
\Sigma_{(1)}^{q q g}\left(p, q, \gamma^{2}, 0\right)= & \Sigma_{(1)}^{q q g}(p, q, 0,0) \\
& +\left[\left[\frac{3}{8} C_{F}+\frac{3}{16} C_{A}\right] \frac{\pi \sqrt{C_{A}} \gamma^{2}}{\mu^{2}}\right.
\end{aligned}
$$

${ }^{*}$ Electronic forms of all the results are contained in an attached data file. 


$$
\begin{aligned}
& +\left[\left[\frac{67}{36}-\frac{4}{9} \psi^{\prime}\left(\frac{1}{3}\right)+\frac{8}{27} \pi^{2}-\frac{2}{3} \ln \left[\frac{C_{A} \gamma^{4}}{\mu^{4}}\right]\right] C_{F}\right. \\
& \left.+\left[\frac{11}{18} \psi^{\prime}\left(\frac{1}{3}\right)-\frac{2941}{1152}-\frac{11}{27} \pi^{2}+\frac{31}{48} \ln \left[\frac{C_{A} \gamma^{4}}{\mu^{4}}\right]\right] C_{A}\right] \frac{C_{A} \gamma^{4}}{\mu^{4}} \\
& \left.+O\left(\frac{\gamma^{6}}{\mu^{6}}\right)\right] a+O\left(a^{2}\right) \\
& \Sigma_{(2)}^{q q g}\left(p, q, \gamma^{2}, 0\right)=\Sigma_{(5)}^{q q g}\left(p, q, \gamma^{2}, 0\right) \\
& =\Sigma_{(2)}^{q q g}(p, q, 0,0) \\
& +\left[-\frac{3 \pi}{16} C_{A} \frac{\sqrt{C_{A}} \gamma^{2}}{\mu^{2}}\right. \\
& +\left[\left[\frac{43}{18}-\frac{8}{9} \psi^{\prime}\left(\frac{1}{3}\right)+\frac{16}{27} \pi^{2}-\frac{5}{6} \ln \left[\frac{C_{A} \gamma^{4}}{\mu^{4}}\right]\right] C_{F}\right. \\
& \left.+\left[\frac{14}{9} \psi^{\prime}\left(\frac{1}{3}\right)-\frac{1351}{288}-\frac{28}{27} \pi^{2}+\frac{325}{192} \ln \left[\frac{C_{A} \gamma^{4}}{\mu^{4}}\right]\right] C_{A}\right] \frac{C_{A} \gamma^{4}}{\mu^{4}} \\
& \left.+O\left(\frac{\gamma^{6}}{\mu^{6}}\right)\right] a+O\left(a^{2}\right) \\
& \Sigma_{(3)}^{q q g}\left(p, q, \gamma^{2}, 0\right)=\Sigma_{(4)}^{q q g}\left(p, q, \gamma^{2}, 0\right) \\
& =\Sigma_{(3)}^{q q g}(p, q, 0,0) \\
& +\left[\left[\frac{3}{16} C_{A}-\frac{3}{4} C_{F}\right] \frac{\pi \sqrt{C_{A}} \gamma^{2}}{\mu^{2}}\right. \\
& +\left[\left[\frac{1}{2} \ln \left[\frac{C_{A} \gamma^{4}}{\mu^{4}}\right]-\frac{4}{3}\right] C_{F}\right. \\
& \left.+\left[\frac{2}{3} \psi^{\prime}\left(\frac{1}{3}\right)-\frac{917}{576}-\frac{4}{9} \pi^{2}+\frac{109}{192} \ln \left[\frac{C_{A} \gamma^{4}}{\mu^{4}}\right]\right] C_{A}\right] \frac{C_{A} \gamma^{4}}{\mu^{4}} \\
& \left.+O\left(\frac{\gamma^{6}}{\mu^{6}}\right)\right] a+O\left(a^{2}\right) \\
& \Sigma_{(6)}^{q q g}\left(p, q, \gamma^{2}, 0\right)=\Sigma_{(6)}^{q q g}(p, q, 0,0) \\
& +\left[\left[\frac{3}{4} C_{F}-\frac{9}{8} C_{A}\right] \frac{\pi \sqrt{C_{A}} \gamma^{2}}{\mu^{2}}\right. \\
& +\left[\left[\frac{31}{18}-\frac{1}{3} \ln \left[\frac{C_{A} \gamma^{4}}{\mu^{4}}\right]\right] C_{F}+\left[\frac{1}{24} \ln \left[\frac{C_{A} \gamma^{4}}{\mu^{4}}\right]-\frac{991}{576}\right] C_{A}\right] \frac{C_{A} \gamma^{4}}{\mu^{4}} \\
& \left.+O\left(\frac{\gamma^{6}}{\mu^{6}}\right)\right] a+O\left(a^{2}\right) \text {. }
\end{aligned}
$$

For the latter the equivalences between various amplitudes merely reflects the underlying leftright symmetry of the vertex in the choice of basis tensors we have used. We have not imposed this symmetry within the computation but instead it has emerged naturally and is regarded as a minor internal check on the programming. Unlike the triple gluon case there is a dimension two correction for all channels of both these vertices. 


\section{$5 \mathcal{Q}$ solution.}

Next we turn to what is termed the $\mathrm{Q}$ solution in the notation used in [29]. In this and subsequent sections the results are more involved due to the presence of an additional mass scale corresponding to the localizing ghost field mass of the particular solution. Therefore, we will have various combinations of $\gamma$ and $\mu_{\mathcal{I}}$. To compactify notation we will define

$$
\mu_{\mathcal{I}_{ \pm}}^{2}=\frac{1}{2}\left[\mu_{\mathcal{I}}^{2} \pm \sqrt{\left[\mu_{\mathcal{I}}^{4}-4 C_{A} \gamma^{4}\right]}\right]
$$

where $\mathcal{I}$ corresponds to the particular solution of interest. For the triple gluon vertex we have the expressions

$$
\begin{aligned}
& \tilde{\Sigma}_{(1)}^{g g g}\left(p, q, \gamma^{2}, \mu_{\mathcal{Q}}^{2}\right)=\tilde{\Sigma}_{(1)}^{g g g}(p, q, 0,0) \\
& +\left[\left[\frac{11}{64} \mu_{\mathcal{Q}}^{2} \sqrt{\left[\mu_{\mathcal{Q}}^{4}-4 C_{A} \gamma^{4}\right]} \ln \left[\frac{\mu_{\mathcal{Q}_{+}}^{2}}{\mu_{\mathcal{Q}_{-}}^{2}}\right]+\frac{3 \mu_{\mathcal{Q}}^{4}}{32} \ln \left[\frac{C_{A} \gamma^{4}}{\mu_{\mathcal{Q}}^{4}}\right]\right.\right. \\
& -\frac{5 \mu_{\mathcal{Q}}^{6}}{64 \sqrt{\left[\mu_{\mathcal{Q}}^{4}-4 C_{A} \gamma^{4}\right]}} \ln \left[\frac{\mu_{\mathcal{Q}_{+}}^{2}}{\mu_{\mathcal{Q}_{-}}^{2}}\right] \\
& \left.+\left[\frac{13}{6}+\frac{7 \pi^{2}}{36}-\frac{7}{24} \psi^{\prime}\left(\frac{1}{3}\right)-\frac{1}{2} \ln \left[\frac{C_{A} \gamma^{4}}{\mu^{4}}\right]\right] C_{A} \gamma^{4}\right] \frac{C_{A}}{\mu^{4}} \\
& \left.+O\left(\frac{\mu_{\mathcal{Q}}^{6}}{\mu^{6}}\right)\right] a+O\left(a^{2}\right) \\
& \tilde{\Sigma}_{(2)}^{g g g}\left(p, q, \gamma^{2}, \mu_{\mathcal{Q}}^{2}\right)=\tilde{\Sigma}_{(2)}^{g g g}(p, q, 0,0) \\
& +\left[\left[\frac{3 \mu_{\mathcal{Q}}^{4}}{64 \sqrt{\left[\mu_{\mathcal{Q}}^{4}-4 C_{A} \gamma^{4}\right]}} \ln \left[\frac{\mu_{\mathcal{Q}_{+}}^{2}}{\mu_{\mathcal{Q}_{-}}^{2}}\right]-\frac{3}{64} \sqrt{\left[\mu_{\mathcal{Q}}^{4}-4 C_{A} \gamma^{4}\right]} \ln \left[\frac{\mu_{\mathcal{Q}_{+}}^{2}}{\mu_{\mathcal{Q}_{-}}^{2}}\right]\right] \frac{C_{A}}{\mu^{2}}\right. \\
& +\left[-\frac{1075}{768} \mu_{\mathcal{Q}}^{2} \sqrt{\left[\mu_{\mathcal{Q}}^{4}-4 C_{A} \gamma^{4}\right]} \ln \left[\frac{\mu_{\mathcal{Q}_{+}}^{2}}{\mu_{\mathcal{Q}_{-}}^{2}}\right]-\frac{3 \mu_{\mathcal{Q}}^{4}}{4} \ln \left[\frac{C_{A} \gamma^{4}}{\mu_{\mathcal{Q}}^{4}}\right]\right. \\
& +\frac{499 \mu_{\mathcal{Q}}^{6}}{768 \sqrt{\left[\mu_{\mathcal{Q}}^{4}-4 C_{A} \gamma^{4}\right]}} \ln \left[\frac{\mu_{\mathcal{Q}_{+}}^{2}}{\mu_{\mathcal{Q}_{-}}^{2}}\right] \\
& \left.\left.+\left[\frac{289}{144}+\frac{787}{192} \ln \left[\frac{C_{A} \gamma^{4}}{\mu^{4}}\right]\right] C_{A} \gamma^{4}\right] \frac{C_{A}}{\mu^{4}}+O\left(\frac{\mu_{\mathcal{Q}}^{6}}{\mu^{6}}\right)\right] a+O\left(a^{2}\right) \\
& \tilde{\Sigma}_{(3)}^{g g g}\left(p, q, \gamma^{2}, \mu_{\mathcal{Q}}^{2}\right)=\tilde{\Sigma}_{(3)}^{g g g}(p, q, 0,0) \\
& +\left[\left[\frac{3 \mu_{\mathcal{Q}}^{4}}{64 \sqrt{\left[\mu_{\mathcal{Q}}^{4}-4 C_{A} \gamma^{4}\right]}} \ln \left[\frac{\mu_{\mathcal{Q}_{+}}^{2}}{\mu_{\mathcal{Q}_{-}}^{2}}\right]-\frac{3}{64} \sqrt{\left[\mu_{\mathcal{Q}}^{4}-4 C_{A} \gamma^{4}\right]} \ln \left[\frac{\mu_{\mathcal{Q}_{+}}^{2}}{\mu_{\mathcal{Q}_{-}}^{2}}\right]\right] \frac{C_{A}}{\mu^{2}}\right. \\
& +\left[-\frac{1087}{768} \mu_{\mathcal{Q}}^{2} \sqrt{\left[\mu_{\mathcal{Q}}^{4}-4 C_{A} \gamma^{4}\right]} \ln \left[\frac{\mu_{\mathcal{Q}_{+}}^{2}}{\mu_{\mathcal{Q}_{-}}^{2}}\right]-\frac{3 \mu_{\mathcal{Q}}^{4}}{4} \ln \left[\frac{C_{A} \gamma^{4}}{\mu_{\mathcal{Q}}^{4}}\right]\right. \\
& +\frac{511 C_{A} \mu_{\mathcal{Q}}^{6}}{768 \sqrt{\left[\mu_{\mathcal{Q}}^{4}-4 C_{A} \gamma^{4}\right]}} \ln \left[\frac{\mu_{\mathcal{Q}_{+}}^{2}}{\mu_{\mathcal{Q}_{-}}^{2}}\right] \\
& \left.+\left[\frac{25}{18} \psi^{\prime}\left(\frac{1}{3}\right)-\frac{2033}{576}-\frac{25 \pi^{2}}{27}+\frac{799}{192} \ln \left[\frac{C_{A} \gamma^{4}}{\mu^{4}}\right]\right] C_{A} \gamma^{4}\right] \frac{C_{A}}{\mu^{4}} \\
& \left.+O\left(\frac{\mu_{\mathcal{Q}}^{6}}{\mu^{6}}\right)\right] a+O\left(a^{2}\right)
\end{aligned}
$$


where like the pure Gribov-Zwanziger case the structure of dimension two corrections are the same. This is somewhat unexpected as the presence of the extra mass does not induce a lower order correction. Therefore, if one were using the absence of a dimension two correction in lattice data in channel 1 , if for example that were the case, then one could not conclude that the pure Gribov-Zwanziger case is an explanation since $\mathcal{Q}$ has the same qualitative feature. We note that here and subsequently we have used $O\left(\mu_{\mathcal{I}}^{6} / \mu^{6}\right)$ to indicate we are dropping dimension six terms and have used $\mu_{\mathcal{I}}^{6}$ within the order symbol to indicate a generic dimension six mass scale. In practice this could be a dependent on $\gamma$ too but it would complicate the notation. For the other two vertices we have

$$
\begin{aligned}
& \Sigma_{(1)}^{c c g}\left(p, q, \gamma^{2}, \mu_{\mathcal{Q}}^{2}\right)=\Sigma_{(1)}^{c c g}(p, q, 0,0) \\
& +\left[\left[-\frac{9 C_{A} \gamma^{4}}{16 \sqrt{\left[\mu_{\mathcal{Q}}^{4}-4 C_{A} \gamma^{4}\right]}} \ln \left[\frac{\mu_{\mathcal{Q}_{+}}^{2}}{\mu_{\mathcal{Q}_{-}}^{2}}\right]\right] \frac{C_{A}}{\mu^{2}}\right. \\
& +\left[\frac{C_{A} \gamma^{4} \mu_{\mathcal{Q}}^{2}}{12 \sqrt{\left[\mu_{\mathcal{Q}}^{4}-4 C_{A} \gamma^{4}\right]}} \ln \left[\frac{\mu_{\mathcal{Q}_{+}}^{2}}{\mu_{\mathcal{Q}_{-}}^{2}}\right]\right. \\
& \left.+\left[\frac{\pi^{2}}{216}-\frac{1}{144} \psi^{\prime}\left(\frac{1}{3}\right)-\frac{377}{2304}+\frac{1}{12} \ln \left[\frac{C_{A} \gamma^{4}}{\mu^{4}}\right]\right] C_{A} \gamma^{4}\right] \frac{C_{A}}{\mu^{4}} \\
& \left.+O\left(\frac{\mu_{\mathcal{Q}}^{6}}{\mu^{6}}\right)\right] a+O\left(a^{2}\right) \\
& \Sigma_{(2)}^{c c g}\left(p, q, \gamma^{2}, \mu_{\mathcal{Q}}^{2}\right)=\Sigma_{(2)}^{c c g}(p, q, 0,0) \\
& +\left[\left[\frac{3 C_{A} \gamma^{4}}{8 \sqrt{\left[\mu_{\mathcal{Q}}^{4}-4 C_{A} \gamma^{4}\right]}} \ln \left[\frac{\mu_{\mathcal{Q}_{+}}^{2}}{\mu_{\mathcal{Q}_{-}}^{2}}\right]\right] \frac{C_{A}}{\mu^{2}}\right. \\
& +\left[-\frac{C_{A} \gamma^{4} \mu_{\mathcal{Q}}^{2}}{48 \sqrt{\left[\mu_{\mathcal{Q}}^{4}-4 C_{A} \gamma^{4}\right]}} \ln \left[\frac{\mu_{\mathcal{Q}_{+}}^{2}}{\mu_{\mathcal{Q}_{-}}^{2}}\right]\right. \\
& \left.+\left[\frac{1}{144} \psi^{\prime}\left(\frac{1}{3}\right)-\frac{\pi^{2}}{216}-\frac{7}{2304}-\frac{1}{48} \ln \left[\frac{C_{A} \gamma^{4}}{\mu^{4}}\right]\right] C_{A} \gamma^{4}\right] \frac{C_{A}}{\mu^{4}} \\
& \left.+O\left(\frac{\mu_{\mathcal{Q}}^{6}}{\mu^{6}}\right)\right] a+O\left(a^{2}\right)
\end{aligned}
$$

and

$$
\begin{aligned}
& \Sigma_{(1)}^{q q g}\left(p, q, \gamma^{2}, \mu_{\mathcal{Q}}^{2}\right)=\Sigma_{(1)}^{q q g}(p, q, 0,0) \\
& +\left[\left[\left[\frac{3}{4} C_{F}+\frac{3}{8} C_{A}\right] \frac{C_{A} \gamma^{4}}{\sqrt{\left[\mu_{\mathcal{Q}}^{4}-4 C_{A} \gamma^{4}\right]}} \ln \left[\frac{\mu_{\mathcal{Q}_{+}}^{2}}{\mu_{\mathcal{Q}_{-}}^{2}}\right]\right] \frac{1}{\mu^{2}}\right. \\
& +\left[\left[-\frac{2 C_{A} \mu_{\mathcal{Q}}^{2} \gamma^{4}}{3 \sqrt{\left[\mu_{\mathcal{Q}}^{4}-4 C_{A} \gamma^{4}\right]}} \ln \left[\frac{\mu_{\mathcal{Q}_{+}}^{2}}{\mu_{\mathcal{Q}_{-}}^{2}}\right]\right.\right. \\
& \left.+\left[\frac{67}{36}+\frac{8 \pi^{2}}{27}-\frac{4}{9} \psi^{\prime}\left(\frac{1}{3}\right)-\frac{2}{3} \ln \left[\frac{C_{A} \gamma^{4}}{\mu^{4}}\right]\right] C_{A} \gamma^{4}\right] C_{F} \\
& +\left[\frac{31 C_{A} \mu_{\mathcal{Q}}^{2} \gamma^{4}}{48 \sqrt{\left[\mu_{\mathcal{Q}}^{4}-4 C_{A} \gamma^{4}\right]}} \ln \left[\frac{\mu_{\mathcal{Q}_{+}}^{2}}{\mu_{\mathcal{Q}_{-}}^{2}}\right]\right.
\end{aligned}
$$




$$
\begin{aligned}
& \left.\left.+\left[\frac{11}{18} \psi^{\prime}\left(\frac{1}{3}\right)-\frac{2941}{1152}-\frac{11 \pi^{2}}{27}+\frac{31}{48} \ln \left[\frac{C_{A} \gamma^{4}}{\mu^{4}}\right]\right] C_{A} \gamma^{4}\right] C_{A}\right] \frac{1}{\mu^{4}} \\
& \left.+O\left(\frac{\mu_{\mathcal{Q}}^{6}}{\mu^{6}}\right)\right] a+O\left(a^{2}\right) \\
& \Sigma_{(2)}^{q q g}\left(p, q, \gamma^{2}, \mu_{\mathcal{Q}}^{2}\right)=\Sigma_{(5)}^{q q g}\left(p, q, \gamma^{2}, \mu_{\mathcal{Q}}^{2}\right) \\
& =\Sigma_{(2)}^{q q g}(p, q, 0,0) \\
& +\left[\left[-\frac{3 C_{A}^{2} \gamma^{4}}{8 \sqrt{\left[\mu_{\mathcal{Q}}^{4}-4 C_{A} \gamma^{4}\right]}} \ln \left[\frac{\mu_{\mathcal{Q}_{+}}^{2}}{\mu_{\mathcal{Q}_{-}}^{2}}\right]\right] \frac{1}{\mu^{2}}\right. \\
& +\left[\left[-\frac{5 C_{A} \mu_{\mathcal{Q}}^{2} \gamma^{4}}{6 \sqrt{\left[\mu_{\mathcal{Q}}^{4}-4 C_{A} \gamma^{4}\right]}} \ln \left[\frac{\mu_{\mathcal{Q}_{+}}^{2}}{\mu_{\mathcal{Q}_{-}}^{2}}\right]\right.\right. \\
& \left.+\left[\frac{43}{18}+\frac{16 \pi^{2}}{27}-\frac{8}{9} \psi^{\prime}\left(\frac{1}{3}\right)-\frac{5}{6} \ln \left[\frac{C_{A} \gamma^{4}}{\mu^{4}}\right]\right] C_{A} \gamma^{4}\right] C_{F} \\
& +\left[\left[\frac{14}{9} \psi^{\prime}\left(\frac{1}{3}\right)-\frac{1351}{288}-\frac{28 \pi^{2}}{27}+\frac{325}{192} \ln \left[\frac{C_{A} \gamma^{4}}{\mu^{4}}\right]\right] C_{A} \gamma^{4}\right. \\
& \left.\left.\left.+\frac{325 C_{A} \mu_{\mathcal{Q}}^{2} \gamma^{4}}{192 \sqrt{\left[\mu_{\mathcal{Q}}^{4}-4 C_{A} \gamma^{4}\right]}} \ln \left[\frac{\mu_{\mathcal{Q}_{+}}^{2}}{\mu_{\mathcal{Q}_{-}}^{2}}\right]\right] C_{A}\right] \frac{1}{\mu^{4}}+O\left(\frac{\mu_{\mathcal{Q}}^{6}}{\mu^{6}}\right)\right] a \\
& +O\left(a^{2}\right) \\
& \Sigma_{(3)}^{q q g}\left(p, q, \gamma^{2}, \mu_{\mathcal{Q}}^{2}\right)=\Sigma_{(4)}^{q q g}\left(p, q, \gamma^{2}, \mu_{\mathcal{Q}}^{2}\right) \\
& =\Sigma_{(3)}^{q q g}(p, q, 0,0) \\
& +\left[\left[\left[\frac{3}{8} C_{A}-\frac{3}{2} C_{F}\right] \frac{C_{A} \gamma^{4}}{\sqrt{\left[\mu_{\mathcal{Q}}^{4}-4 C_{A} \gamma^{4}\right]}} \ln \left[\frac{\mu_{\mathcal{Q}_{+}}^{2}}{\mu_{\mathcal{Q}_{-}}^{2}}\right]\right] \frac{1}{\mu^{2}}\right. \\
& +\left[\left[\frac{C_{A} \mu_{\mathcal{Q}}^{2} \gamma^{4}}{2 \sqrt{\left[\mu_{\mathcal{Q}}^{4}-4 C_{A} \gamma^{4}\right]}} \ln \left[\frac{\mu_{\mathcal{Q}_{+}}^{2}}{\mu_{\mathcal{Q}_{-}}^{2}}\right]+\left[\frac{1}{2} \ln \left[\frac{C_{A} \gamma^{4}}{\mu^{4}}\right]-\frac{4}{3}\right] C_{A} \gamma^{4}\right] C_{F}\right. \\
& +\left[\frac{109 C_{A} \mu_{\mathcal{Q}}^{2} \gamma^{4}}{192 \sqrt{\left[\mu_{\mathcal{Q}}^{4}-4 C_{A} \gamma^{4}\right]}} \ln \left[\frac{\mu_{\mathcal{Q}_{+}}^{2}}{\mu_{\mathcal{Q}_{-}}^{2}}\right]\right. \\
& \left.\left.+\left[\frac{2}{3} \psi^{\prime}\left(\frac{1}{3}\right)-\frac{917}{576}-\frac{4 \pi^{2}}{9}+\frac{109}{192} \ln \left[\frac{C_{A} \gamma^{4}}{\mu^{4}}\right]\right] C_{A} \gamma^{4}\right] C_{A}\right] \frac{1}{\mu^{4}} \\
& \left.+O\left(\frac{\mu_{\mathcal{Q}}^{6}}{\mu^{6}}\right)\right] a+O\left(a^{2}\right) \\
& \Sigma_{(6)}^{q q g}\left(p, q, \gamma^{2}, \mu_{\mathcal{Q}}^{2}\right)=\Sigma_{(6)}^{q q g}(p, q, 0,0) \\
& +\left[\left[\left[\frac{3}{2} C_{F}-\frac{9}{4} C_{A}\right] \frac{C_{A} \gamma^{4}}{\sqrt{\left[\mu_{\mathcal{Q}}^{4}-4 C_{A} \gamma^{4}\right]}} \ln \left[\frac{\mu_{\mathcal{Q}_{+}}^{2}}{\mu_{\mathcal{Q}_{-}}^{2}}\right]\right] \frac{1}{\mu^{2}}\right. \\
& +\left[\left[\left[\frac{31}{18}-\frac{1}{3} \ln \left[\frac{C_{A} \gamma^{4}}{\mu^{4}}\right]\right] C_{A} \gamma^{4}-\frac{C_{A} \mu_{\mathcal{Q}}^{2} \gamma^{4}}{3 \sqrt{\left[\mu_{\mathcal{Q}}^{4}-4 C_{A} \gamma^{4}\right]}} \ln \left[\frac{\mu_{\mathcal{Q}_{+}}^{2}}{\mu_{\mathcal{Q}_{-}}^{2}}\right]\right] C_{F}\right.
\end{aligned}
$$




$$
\begin{aligned}
& +\left[\frac{C_{A} \mu_{\mathcal{Q}}^{2} \gamma^{4}}{24 \sqrt{\left[\mu_{\mathcal{Q}}^{4}-4 C_{A} \gamma^{4}\right]}} \ln \left[\frac{\mu_{\mathcal{Q}_{+}}^{2}}{\mu_{\mathcal{Q}_{-}}^{2}}\right]\right. \\
& \left.\left.+\left[\frac{1}{24} \ln \left[\frac{C_{A} \gamma^{4}}{\mu^{4}}\right]-\frac{991}{576}\right] C_{A} \gamma^{4}\right] C_{A}\right] \frac{1}{\mu^{4}} \\
& \left.+O\left(\frac{\mu_{\mathcal{Q}}^{6}}{\mu^{6}}\right)\right] a+O\left(a^{2}\right) .
\end{aligned}
$$

Again these expressions all have dimension two corrections albeit complicated. However, they have the same qualitative structure as the pure Gribov-Zwanziger case. In the above expressions for all the amplitudes we have checked that the results of the previous section are reproduced as $\mu_{\mathcal{Q}}^{2} \rightarrow 0$. This also applies to results in subsequent sections in the appropriate $\mu_{\mathcal{I}}^{2} \rightarrow 0$ limit. We have also checked that in the $\gamma^{2} \rightarrow 0$ limit there are no corrections at all as there should be since then there is no horizon condition.

\section{$6 \mathcal{R}$ solution.}

Structurally, the expressions for the $\mathcal{R}$ are very similar to those for $\mathcal{Q}$. For instance, in many cases the differences are only in the numerical coefficients. Though the mass is $\mu_{\mathcal{R}}$ rather than $\mu_{\mathcal{Q}}$ of course. Therefore, we will make minimal comment on these parallel results. For the triple gluon vertex we have,

$$
\begin{aligned}
& \tilde{\Sigma}_{(1)}^{g g g}\left(p, q, \gamma^{2}, \mu_{\mathcal{R}}^{2}\right)=\tilde{\Sigma}_{(1)}^{g g g}(p, q, 0,0) \\
& +\left[\left[\frac{3}{32} \mu_{\mathcal{R}}^{2} \sqrt{\left[\mu_{\mathcal{R}}^{4}-4 C_{A} \gamma^{4}\right]} \ln \left[\frac{\mu_{\mathcal{R}_{+}}^{2}}{\mu_{\mathcal{R}_{-}}^{2}}\right]+\frac{3 \mu_{\mathcal{R}}^{4}}{32} \ln \left[\frac{C_{A} \gamma^{4}}{\mu_{\mathcal{R}}^{4}}\right]\right.\right. \\
& -\frac{5 C_{A} \mu_{\mathcal{R}}^{2} \gamma^{4}}{16 \sqrt{\left[\mu_{\mathcal{R}}^{4}-4 C_{A} \gamma^{4}\right]}} \ln \left[\frac{\mu_{\mathcal{R}_{+}}^{2}}{\mu_{\mathcal{R}_{-}}^{2}}\right] \\
& \left.+\left[\frac{13}{6}+\frac{7 \pi^{2}}{36}-\frac{7}{24} \psi^{\prime}\left(\frac{1}{3}\right)-\frac{1}{2} \ln \left[\frac{C_{A} \gamma^{4}}{\mu^{4}}\right]\right] C_{A} \gamma^{4}\right] \frac{C_{A}}{\mu^{4}} \\
& \left.+O\left(\frac{\mu_{\mathcal{R}}^{6}}{\mu^{6}}\right)\right] a+O\left(a^{2}\right) \\
& \tilde{\Sigma}_{(2)}^{g g g}\left(p, q, \gamma^{2}, \mu_{\mathcal{R}}^{2}\right)=\tilde{\Sigma}_{(2)}^{g g g}(p, q, 0,0) \\
& +\left[\left[\frac{3 C_{A} \gamma^{4}}{16 \sqrt{\left[\mu_{\mathcal{R}}^{4}-4 C_{A} \gamma^{4}\right]}} \ln \left[\frac{\mu_{\mathcal{R}_{+}}^{2}}{\mu_{\mathcal{R}_{-}}^{2}}\right]\right] \frac{C_{A}}{\mu^{2}}\right. \\
& +\left[-\frac{3}{4} \mu_{\mathcal{R}}^{2} \sqrt{\left[\mu_{\mathcal{R}}^{4}-4 C_{A} \gamma^{4}\right]} \ln \left[\frac{\mu_{\mathcal{R}_{+}}^{2}}{\mu_{\mathcal{R}_{-}}^{2}}\right]-\frac{3 \mu_{\mathcal{R}}^{4}}{4} \ln \left[\frac{C_{A} \gamma^{4}}{\mu_{\mathcal{R}}^{4}}\right]\right. \\
& +\frac{499 C_{A} \mu_{\mathcal{R}}^{2} \gamma^{4}}{192 \sqrt{\left[\mu_{\mathcal{R}}^{4}-4 C_{A} \gamma^{4}\right]}} \ln \left[\frac{\mu_{\mathcal{R}_{+}}^{2}}{\mu_{\mathcal{R}_{-}}^{2}}\right] \\
& \left.\left.+\left[\frac{289}{144}+\frac{787}{192} \ln \left[\frac{C_{A} \gamma^{4}}{\mu^{4}}\right]\right] C_{A} \gamma^{4}\right] \frac{C_{A}}{\mu^{4}}+O\left(\frac{\mu_{\mathcal{R}}^{6}}{\mu^{6}}\right)\right] a+O\left(a^{2}\right) \\
& \tilde{\Sigma}_{(3)}^{g g g}\left(p, q, \gamma^{2}, \mu_{\mathcal{R}}^{2}\right)=\tilde{\Sigma}_{(3)}^{g g g}(p, q, 0,0)
\end{aligned}
$$




$$
\begin{aligned}
& +\left[\left[\frac{3 C_{A} \gamma^{4}}{16 \sqrt{\left[\mu_{\mathcal{R}}^{4}-4 C_{A} \gamma^{4}\right]}} \ln \left[\frac{\mu_{\mathcal{R}_{+}}^{2}}{\mu_{\mathcal{R}_{-}}^{2}}\right]\right] \frac{C_{A}}{\mu^{2}}\right. \\
& +\left[-\frac{3}{4} \mu_{\mathcal{R}}^{2} \sqrt{\left[\mu_{\mathcal{R}}^{4}-4 C_{A} \gamma^{4}\right]} \ln \left[\frac{\mu_{\mathcal{R}_{+}}^{2}}{\mu_{\mathcal{R}_{-}}^{2}}\right]-\frac{3 \mu_{\mathcal{R}}^{4}}{4} \ln \left[\frac{C_{A} \gamma^{4}}{\mu_{\mathcal{R}}^{4}}\right]\right. \\
& +\frac{511 C_{A} \mu_{\mathcal{R}}^{2} \gamma^{4}}{192 \sqrt{\left[\mu_{\mathcal{R}}^{4}-4 C_{A} \gamma^{4}\right]}} \ln \left[\frac{\mu_{\mathcal{R}_{+}}^{2}}{\mu_{\mathcal{R}_{-}}^{2}}\right] \\
& \left.+\left[\frac{25}{18} \psi^{\prime}\left(\frac{1}{3}\right)-\frac{2033}{576}-\frac{25 \pi^{2}}{27}+\frac{799}{192} \ln \left[\frac{C_{A} \gamma^{4}}{\mu^{4}}\right]\right] C_{A} \gamma^{4}\right] \frac{C_{A}}{\mu^{4}} \\
& \left.+O\left(\frac{\mu_{\mathcal{R}}^{6}}{\mu^{6}}\right)\right] a+O\left(a^{2}\right) \text {. }
\end{aligned}
$$

Again there is no dimension two correction for channel 1. For the other two vertices it transpires that aside from mapping the masses $\mu_{\mathcal{Q}} \leftrightarrow \mu_{\mathcal{R}}$ the expressions for the ghost-gluon and quarkgluon vertices are formally the same at one loop. This is not unexpected if one considers the contributing Feynman diagrams. The propagators for $\mathcal{Q}$ and $\mathcal{R}$ differ only in the localizing ghost sector. Since the gluon propagator is the only propagator from the gauge sector which contributes to the ghost-gluon and quark-gluon vertices at one loop and is formally the same for both solutions then the respective vertex functions have to be the same.

\section{Explicit gluon mass.}

So far we have concentrated on Gribov and Stingl type propagators for the gluon sector of YangMills based on the pure and refined Gribov-Zwanziger Lagrangians. However, an alternative scenario is that a frozen gluon propagator in the infrared could be as a result of a pure gluon mass. While such a term ordinarily breaks gauge invariance, it is possible to construct a gauge invariant mass operator for the gluon. However, such an operator has to be non-local, [32, 33, and could be associated with an analogous ghost operator which is non-local. The origin for the latter observation is that it is possible to have a BRST invariant gluon mass term which was considered in [50]. There in order to make that local gluon mass operator BRST invariant one had to include a ghost mass term which was dependent on the gauge parameter. In either case when one restricts both to the Landau gauge the non-locality disappears in the first case and in the second only one of the two terms contributing to the BRST operator survives. In both cases in the Landau gauge

$$
\mathcal{O}_{A_{\mu}^{2}}=\frac{\mu_{\mathcal{X}}^{2}}{2} A_{\mu}^{a} A^{a \mu}
$$

emerges as the mass term for the gluon. We use the same notation as 30] for consistency and will regard the presence of (7.1) in a Lagrangian as our control calculation. With (7.1) we can derive the propagator for $A_{\mu}^{a}$. However, in order to compare with the previous two cases we will also add (7.1) into the Gribov-Zwanziger Lagrangian. Thus our propagators are

$$
\begin{aligned}
\left\langle A_{\mu}^{a}(p) A_{\nu}^{b}(-p)\right\rangle_{\mathcal{X}} & =-\frac{\delta^{a b} p^{2}}{\left[\left(p^{2}\right)^{2}+\mu_{\mathcal{X}}^{2} p^{2}+C_{A} \gamma^{4}\right]} P_{\mu \nu}(p) \\
\left\langle A_{\mu}^{a}(p) \xi_{\nu}^{b c}(-p)\right\rangle_{\mathcal{X}} & =\frac{i f^{a b c} \gamma^{2}}{\left[\left(p^{2}\right)^{2}+\mu_{\mathcal{X}}^{2} p^{2}+C_{A} \gamma^{4}\right]} P_{\mu \nu}(p) \\
\left\langle A_{\mu}^{a}(p) \rho_{\nu}^{b c}(-p)\right\rangle_{\mathcal{X}} & =0,\left\langle\xi_{\mu}^{a b}(p) \rho_{\nu}^{c d}(-p)\right\rangle_{\mathcal{X}}=0
\end{aligned}
$$




$$
\begin{aligned}
\left\langle\xi_{\mu}^{a b}(p) \xi_{\nu}^{c d}(-p)\right\rangle_{\mathcal{X}} & =-\frac{\delta^{a c} \delta^{b d}}{p^{2}} \eta_{\mu \nu}+\frac{f^{a b e} f^{c d e} \gamma^{4}}{p^{2}\left[\left(p^{2}\right)^{2}+\mu_{\mathcal{X}}^{2} p^{2}+C_{A} \gamma^{4}\right]} P_{\mu \nu}(p) \\
\left\langle\rho_{\mu}^{a b}(p) \rho_{\nu}^{c d}(-p)\right\rangle_{\mathcal{X}} & =\left\langle\omega_{\mu}^{a b}(p) \bar{\omega}_{\nu}^{c d}(-p)\right\rangle_{\mathcal{X}}=-\frac{\delta^{a c} \delta^{b d}}{p^{2}} \eta_{\mu \nu} .
\end{aligned}
$$

Clearly in the limit where $\gamma \rightarrow 0$ the first term reduces to what one expects for a gluon propagator with a naive mass term. Although the $\rho_{\mu}^{a b}, \xi_{\mu}^{a b}$ and $\omega_{\mu}^{a b}$ propagators remain their contribution cancels within any calculation as if they were not present in the first case in this limit. Though for any results we present we have checked this directly by using the usual Yang-Mills Lagrangian without including a Gribov mass term.

The procedure to compute the vertex functions for this case is precisely the same as the previous two sections. However, we first give the results for a pure mass term, $\mu_{\mathcal{X}}$, in the absence of $\gamma$. For the triple gluon vertex we have

$$
\begin{aligned}
\tilde{\Sigma}_{(1)}^{g g g}\left(p, q, 0, \mu_{\mathcal{X}}^{2}\right)= & \tilde{\Sigma}_{(1)}^{g g g}(p, q, 0,0) \\
+ & {\left[\left[\frac{7}{4}+\frac{14 \pi^{2}}{27}-\frac{7}{9} \psi^{\prime}\left(\frac{1}{3}\right)\right] \frac{\mu_{\mathcal{X}}^{2}}{\mu^{2}}\right.} \\
& \left.+\left[\frac{5 \pi^{2}}{27}-\frac{17}{12}-\frac{5}{18} \psi^{\prime}\left(\frac{1}{3}\right)+\frac{49}{16} \ln \left[\frac{\mu_{\mathcal{X}}^{2}}{\mu^{2}}\right]\right] \frac{\mu_{\mathcal{X}}^{4}}{\mu^{4}}+O\left(\frac{\mu_{\mathcal{X}}^{6}}{\mu^{6}}\right)\right] C_{A} a \\
+ & O\left(a^{2}\right) \\
\tilde{\Sigma}_{(2)}^{g g g}\left(p, q, 0, \mu_{\mathcal{X}}^{2}\right)= & \tilde{\Sigma}_{(2)}^{g g g}(p, q, 0,0) \\
+ & {\left[\left[\frac{673}{96}-\frac{4 \pi^{2}}{27}+\frac{2}{9} \psi^{\prime}\left(\frac{1}{3}\right)-\frac{3}{16} \ln \left[\frac{\mu_{\mathcal{X}}^{2}}{\mu^{2}}\right]\right] \frac{\mu_{\mathcal{X}}^{2}}{\mu^{2}}\right.} \\
& \left.+\left[\frac{209}{36}-\frac{26}{3} \ln \left[\frac{\mu_{\mathcal{X}}^{2}}{\mu^{2}}\right]\right] \frac{\mu_{\mathcal{X}}^{4}}{\mu^{4}}+O\left(\frac{\mu_{\mathcal{X}}^{6}}{\mu^{6}}\right)\right] C_{A} a+O\left(a^{2}\right) \\
\tilde{\Sigma}_{(3)}^{g g g}\left(p, q, 0, \mu_{\mathcal{X}}^{2}\right)= & {\left[\left[\frac{596}{96}-\frac{4 \pi^{2}}{9}+\frac{2}{3} \psi^{\prime}\left(\frac{1}{3}\right)-\frac{3}{16} \ln \left[\frac{\mu_{\mathcal{X}}^{2}}{\mu^{2}}\right]\right] \frac{\mu_{\mathcal{X}}^{2}}{\mu^{2}}\right.} \\
+ & \left.+\left[\frac{433}{72}-\frac{8 \pi^{2}}{27}+\frac{4}{9} \psi^{\prime}\left(\frac{1}{3}\right)-\frac{211}{24} \ln \left[\frac{\mu_{\mathcal{X}}^{2}}{\mu^{2}}\right]\right] \frac{\mu_{\mathcal{X}}^{4}}{\mu^{4}}+O\left(\frac{\mu_{\mathcal{X}}^{6}}{\mu^{6}}\right)\right] C_{A^{a}} \\
+ & O\left(a^{2}\right) .
\end{aligned}
$$

In contrast to the pure Gribov-Zwanziger case and the $\mathcal{Q}$ and $\mathcal{R}$ extensions there is a dimension two correction in channel 1 as well as the other two channels. This is a significant departure from the three Gribov scenarios and will form part of our test. For the other two vertices we have

$$
\begin{aligned}
\Sigma_{(1)}^{c c g}\left(p, q, 0, \mu_{\mathcal{X}}^{2}\right)= & \Sigma_{(1)}^{c c g}(p, q, 0,0) \\
+ & {\left[\left[\frac{1}{36} \psi^{\prime}\left(\frac{1}{3}\right)-\frac{11}{32}-\frac{\pi^{2}}{54}+\frac{9}{16} \ln \left[\frac{\mu_{\mathcal{X}}^{2}}{\mu^{2}}\right]\right] \frac{\mu_{\mathcal{X}}^{2}}{\mu^{2}}\right.} \\
& \left.+\left[\frac{83}{576}-\frac{\pi^{2}}{54}+\frac{1}{36} \psi^{\prime}\left(\frac{1}{3}\right)+\frac{5}{24} \ln \left[\frac{\mu_{\mathcal{X}}^{2}}{\mu^{2}}\right]\right] \frac{\mu_{\mathcal{X}}^{4}}{\mu^{4}}+O\left(\frac{\mu_{\mathcal{X}}^{6}}{\mu^{6}}\right)\right] C_{A} a \\
& +O\left(a^{2}\right) \\
\Sigma_{(2)}^{c c g}\left(p, q, 0, \mu_{\mathcal{X}}^{2}\right)= & \Sigma_{(2)}^{c c g}(p, q, 0,0)
\end{aligned}
$$




$$
\begin{aligned}
& +\left[\left[\frac{1}{8}-\frac{\pi^{2}}{27}+\frac{1}{18} \psi^{\prime}\left(\frac{1}{3}\right)-\frac{3}{8} \ln \left[\frac{\mu_{\mathcal{X}}^{2}}{\mu^{2}}\right]\right] \frac{\mu_{\mathcal{X}}^{2}}{\mu^{2}}\right. \\
& +\left[\frac{13}{576}+\frac{\pi^{2}}{54}-\frac{1}{36} \psi^{\prime}\left(\frac{1}{3}\right)-\frac{1}{3} \ln \left[\frac{\mu_{\mathcal{X}}^{2}}{\mu^{2}}\right]\right] \frac{\mu_{\mathcal{X}}^{4}}{\mu^{4}} \\
& \left.+O\left(\frac{\mu_{\mathcal{X}}^{6}}{\mu^{6}}\right)\right] C_{A} a+O\left(a^{2}\right)
\end{aligned}
$$

and

$$
\begin{aligned}
& \Sigma_{(1)}^{q q g}\left(p, q, 0, \mu_{\mathcal{X}}^{2}\right)=\Sigma_{(1)}^{q q g}(p, q, 0,0) \\
& +\left[\left[\left[\frac{13}{18} \psi^{\prime}\left(\frac{1}{3}\right)+\frac{5}{16}-\frac{13 \pi^{2}}{27}-\frac{3}{8} \ln \left[\frac{\mu_{\mathcal{X}}^{2}}{\mu^{2}}\right]\right] C_{A}\right.\right. \\
& \left.+\left[\frac{7}{8}-\frac{5}{9} \psi^{\prime}\left(\frac{1}{3}\right)+\frac{10 \pi^{2}}{27}-\frac{3}{4} \ln \left[\frac{\mu_{\mathcal{X}}^{2}}{\mu^{2}}\right]\right] C_{F}\right] \frac{\mu_{\mathcal{X}}^{2}}{\mu^{2}} \\
& +\left[\left[\frac{661}{288}+\frac{8 \pi^{2}}{27}-\frac{4}{9} \psi^{\prime}\left(\frac{1}{3}\right)-\frac{49}{24} \ln \left[\frac{\mu_{\mathcal{X}}^{2}}{\mu^{2}}\right]\right] C_{A}\right. \\
& \left.\left.+\left[\frac{4}{9} \psi^{\prime}\left(\frac{1}{3}\right)-\frac{67}{36}-\frac{8 \pi^{2}}{27}+\frac{4}{3} \ln \left[\frac{\mu_{\mathcal{X}}^{2}}{\mu^{2}}\right]\right] C_{F}\right] \frac{\mu_{\mathcal{X}}^{4}}{\mu^{4}}+O\left(\frac{\mu_{\mathcal{X}}^{6}}{\mu^{6}}\right)\right] a \\
& +O\left(a^{2}\right) \\
& \Sigma_{(2)}^{q q g}\left(p, q, 0, \mu_{\mathcal{X}}^{2}\right)=\Sigma_{(5)}^{q q g}\left(p, q, 0, \mu_{\mathcal{X}}^{2}\right) \\
& =\Sigma_{(2)}^{q q g}(p, q, 0,0) \\
& +\left[\left[\left[\frac{8}{9} \psi^{\prime}\left(\frac{1}{3}\right)+\frac{7}{48}-\frac{16 \pi^{2}}{27}+\frac{3}{8} \ln \left[\frac{\mu_{\mathcal{X}}^{2}}{\mu^{2}}\right]\right] C_{A}\right.\right. \\
& \left.+\left[\frac{5}{3}-\frac{8}{9} \psi^{\prime}\left(\frac{1}{3}\right)+\frac{16 \pi^{2}}{27}\right] C_{F}\right] \frac{\mu_{\mathcal{X}}^{2}}{\mu^{2}} \\
& +\left[\left[\frac{295}{72}+\frac{16 \pi^{2}}{27}-\frac{8}{9} \psi^{\prime}\left(\frac{1}{3}\right)-\frac{79}{24} \ln \left[\frac{\mu_{\mathcal{X}}^{2}}{\mu^{2}}\right]\right] C_{A}\right. \\
& \left.\left.+\left[\frac{8}{9} \psi^{\prime}\left(\frac{1}{3}\right)-\frac{43}{18}-\frac{16 \pi^{2}}{27}+\frac{5}{3} \ln \left[\frac{\mu_{\mathcal{X}}^{2}}{\mu^{2}}\right]\right] C_{F}\right] \frac{\mu_{\mathcal{X}}^{4}}{\mu^{4}}+O\left(\frac{\mu_{\mathcal{X}}^{6}}{\mu^{6}}\right)\right] a \\
& +O\left(a^{2}\right) \\
& \Sigma_{(3)}^{q q g}\left(p, q, 0, \mu_{\mathcal{X}}^{2}\right)=\Sigma_{(4)}^{q q g}\left(p, q, 0, \mu_{\mathcal{X}}^{2}\right) \\
& =\Sigma_{(3)}^{q q g}(p, q, 0,0) \\
& +\left[\left[\left[\frac{2}{9} \psi^{\prime}\left(\frac{1}{3}\right)+\frac{29}{48}-\frac{4 \pi^{2}}{27}-\frac{3}{8} \ln \left[\frac{\mu_{\mathcal{X}}^{2}}{\mu^{2}}\right]\right] C_{A}\right.\right. \\
& \left.+\left[\frac{19}{12}-\frac{2}{9} \psi^{\prime}\left(\frac{1}{3}\right)+\frac{4 \pi^{2}}{27}+\frac{3}{2} \ln \left[\frac{\mu_{\mathcal{X}}^{2}}{\mu^{2}}\right]\right] C_{F}\right] \frac{\mu_{\mathcal{X}}^{2}}{\mu^{2}} \\
& +\left[\left[\frac{65}{144}-\frac{25}{24} \ln \left[\frac{\mu_{\mathcal{X}}^{2}}{\mu^{2}}\right]\right] C_{A}\right. \\
& \left.\left.+\left[\frac{4}{3}-\ln \left[\frac{\mu_{\mathcal{X}}^{2}}{\mu^{2}}\right]\right] C_{F}\right] \frac{\mu_{\mathcal{X}}^{4}}{\mu^{4}}+O\left(\frac{\mu_{\mathcal{X}}^{6}}{\mu^{6}}\right)\right] a+O\left(a^{2}\right)
\end{aligned}
$$

$\Sigma_{(6)}^{q q g}\left(p, q, 0, \mu_{\mathcal{X}}^{2}\right)=\Sigma_{(6)}^{q q g}(p, q, 0,0)$ 


$$
\begin{aligned}
+\left[\left[\left[\frac{7}{9} \psi^{\prime}\left(\frac{1}{3}\right)-\frac{27}{8}-\frac{14 \pi^{2}}{27}+\frac{9}{4} \ln \left[\frac{\mu_{\mathcal{X}}^{2}}{\mu^{2}}\right]\right] C_{A}\right.\right. \\
\left.+\left[\frac{7}{4}-\frac{2}{3} \psi^{\prime}\left(\frac{1}{3}\right)+\frac{4 \pi^{2}}{9}-\frac{3}{2} \ln \left[\frac{\mu_{\mathcal{X}}^{2}}{\mu^{2}}\right]\right] C_{F}\right] \frac{\mu_{\mathcal{X}}^{2}}{\mu^{2}} \\
+\left[\left[\frac{223}{144}+\frac{17}{12} \ln \left[\frac{\mu_{\mathcal{X}}^{2}}{\mu^{2}}\right]\right] C_{A}\right. \\
\left.\left.+\left[\frac{2}{3} \ln \left[\frac{\mu_{\mathcal{X}}^{2}}{\mu^{2}}\right]-\frac{31}{18}\right] C_{F}\right] \frac{\mu_{\mathcal{X}}^{4}}{\mu^{4}}+O\left(\frac{\mu_{\mathcal{X}}^{6}}{\mu^{6}}\right)\right] a+O\left(a^{2}\right) .
\end{aligned}
$$

Both these cases are completely parallel to earlier sections.

Considering the situation where there is a Gribov mass as well as a gluon mass term we have

$$
\begin{aligned}
& \tilde{\Sigma}_{(1)}^{g g g}\left(p, q, \gamma^{2}, \mu_{\mathcal{X}}^{2}\right)=\tilde{\Sigma}_{(1)}^{g g g}(p, q, 0,0) \\
& +\left[\left[\frac{7}{4}+\frac{14 \pi^{2}}{27}-\frac{7}{9} \psi^{\prime}\left(\frac{1}{3}\right)\right] \frac{C_{A} \mu_{\mathcal{X}}^{2}}{\mu^{2}}\right. \\
& +\left[\frac{57}{64} \mu_{\mathcal{X}}^{2} \sqrt{\left[\mu_{\mathcal{X}}^{4}-4 C_{A} \gamma^{4}\right]} \ln \left[\frac{\mu_{\mathcal{X}_{+}}^{2}}{\mu_{\mathcal{X}_{-}}^{2}}\right]\right. \\
& +\left[\frac{5 \pi^{2}}{27}-\frac{17}{12}-\frac{5}{18} \psi^{\prime}\left(\frac{1}{3}\right)+\frac{49}{32} \ln \left[\frac{C_{A} \gamma^{4}}{\mu^{4}}\right]\right] \mu_{\mathcal{X}}^{4} \\
& +\frac{41 \mu_{\mathcal{X}}^{6}}{64 \sqrt{\left[\mu_{\mathcal{X}}^{4}-4 C_{A} \gamma^{4}\right]}} \ln \left[\frac{\mu_{\mathcal{X}_{+}}^{2}}{\mu_{\mathcal{X}_{-}}^{2}}\right] \\
& \left.+\left[\frac{13}{6}+\frac{7 \pi^{2}}{36}-\frac{7}{24} \psi^{\prime}\left(\frac{1}{3}\right)-\frac{1}{2} \ln \left[\frac{C_{A} \gamma^{4}}{\mu^{4}}\right]\right] C_{A} \gamma^{4}\right] \frac{C_{A}}{\mu^{4}} \\
& \left.+O\left(\frac{\mu_{\mathcal{X}}^{6}}{\mu^{6}}\right)\right] a+O\left(a^{2}\right) \\
& \tilde{\Sigma}_{(2)}^{g g g}\left(p, q, \gamma^{2}, \mu_{\mathcal{X}}^{2}\right)=\tilde{\Sigma}_{(2)}^{g g g}(p, q, 0,0) \\
& +\left[\left[\left[\frac{673}{96}-\frac{4 \pi^{2}}{27}+\frac{2}{9} \psi^{\prime}\left(\frac{1}{3}\right)-\frac{3}{32} \ln \left[\frac{C_{A} \gamma^{4}}{\mu^{4}}\right]\right] \mu_{\mathcal{X}}^{2}\right.\right. \\
& -\frac{3 \mu_{\mathcal{X}}^{4}}{64 \sqrt{\left[\mu_{\mathcal{X}}^{4}-4 C_{A} \gamma^{4}\right]}} \ln \left[\frac{\mu_{\mathcal{X}_{+}}^{2}}{\mu_{\mathcal{X}_{-}}^{2}}\right] \\
& \left.-\frac{3}{64} \sqrt{\left[\mu_{\mathcal{X}}^{4}-4 C_{A} \gamma^{4}\right]} \ln \left[\frac{\mu_{\mathcal{X}_{+}}^{2}}{\mu_{\mathcal{X}_{-}}^{2}}\right]\right] \frac{C_{A}}{\mu^{2}} \\
& +\left[-\frac{817}{256} \mu_{\mathcal{X}}^{2} \sqrt{\left[\mu_{\mathcal{X}}^{4}-4 C_{A} \gamma^{4}\right]} \ln \left[\frac{\mu_{\mathcal{X}_{+}}^{2}}{\mu_{\mathcal{X}_{-}}^{2}}\right]+\left[\frac{209}{36}-\frac{13}{3} \ln \left[\frac{C_{A} \gamma^{4}}{\mu^{4}}\right]\right] \mu_{\mathcal{X}}^{4}\right. \\
& -\frac{877 \mu_{\mathcal{X}}^{6}}{768 \sqrt{\left[\mu_{\mathcal{X}}^{4}-4 C_{A} \gamma^{4}\right]}} \ln \left[\frac{\mu_{\mathcal{X}_{+}}^{2}}{\mu_{\mathcal{X}_{-}}^{2}}\right] \\
& \left.\left.+\left[\frac{289}{144}+\frac{787}{192} \ln \left[\frac{C_{A} \gamma^{4}}{\mu^{4}}\right]\right] C_{A} \gamma^{4}\right] \frac{C_{A}}{\mu^{4}}+O\left(\frac{\mu_{\mathcal{X}}^{6}}{\mu^{6}}\right)\right] a \\
& +O\left(a^{2}\right) \\
& \tilde{\Sigma}_{(3)}^{g g g}\left(p, q, \gamma^{2}, \mu_{\mathcal{X}}^{2}\right)=\tilde{\Sigma}_{(3)}^{g g g}(p, q, 0,0)
\end{aligned}
$$




$$
\begin{aligned}
& +\left[\left[\left[\frac{596}{96}-\frac{4 \pi^{2}}{9}+\frac{2}{3} \psi^{\prime}\left(\frac{1}{3}\right)-\frac{3}{32} \ln \left[\frac{C_{A} \gamma^{4}}{\mu^{4}}\right]\right] \mu_{\mathcal{X}}^{2}\right.\right. \\
& -\frac{3 \mu_{\mathcal{X}}^{4}}{64 \sqrt{\left[\mu_{\mathcal{X}}^{4}-4 C_{A} \gamma^{4}\right]}} \ln \left[\frac{\mu_{\mathcal{X}_{+}}^{2}}{\mu_{\mathcal{X}_{-}}^{2}}\right] \\
& \left.-\frac{3}{64} \sqrt{\left[\mu_{\mathcal{X}}^{4}-4 C_{A} \gamma^{4}\right]} \ln \left[\frac{\mu_{\mathcal{X}_{+}}^{2}}{\mu_{\mathcal{X}_{-}}^{2}}\right]\right] \frac{C_{A}}{\mu^{2}} \\
& +\left[-\frac{829}{256} \mu_{\mathcal{X}}^{2} \sqrt{\left[\mu_{\mathcal{X}}^{4}-4 C_{A} \gamma^{4}\right]} \ln \left[\frac{\mu_{\mathcal{X}_{+}}^{2}}{\mu_{\mathcal{X}_{-}}^{2}}\right]\right. \\
& +\left[\frac{433}{72}-\frac{8 \pi^{2}}{27}+\frac{4}{9} \psi^{\prime}\left(\frac{1}{3}\right)-\frac{211}{48} \ln \left[\frac{C_{A} \gamma^{4}}{\mu^{4}}\right]\right] \mu_{\mathcal{X}}^{4} \\
& -\frac{889 \mu_{\mathcal{X}}^{6}}{768 \sqrt{\left[\mu_{\mathcal{X}}^{4}-4 C_{A} \gamma^{4}\right]}} \ln \left[\frac{\mu_{\mathcal{X}_{+}}^{2}}{\mu_{\mathcal{X}_{-}}^{2}}\right] \\
& \left.+\left[\frac{25}{18} \psi^{\prime}\left(\frac{1}{3}\right)-\frac{2033}{576}-\frac{25 \pi^{2}}{27}+\frac{799}{192} \ln \left[\frac{C_{A} \gamma^{4}}{\mu^{4}}\right]\right] C_{A} \gamma^{4}\right] \frac{C_{A}}{\mu^{4}} \\
& \left.+O\left(\frac{\mu_{\mathcal{X}}^{6}}{\mu^{6}}\right)\right] a+O\left(a^{2}\right)
\end{aligned}
$$

for the triple gluon vertex. The dimension two contribution in channel 1 remains as the leading correction but interestingly it is independent of $\gamma$. This is not the case for the other two channels in that the dimension two term derives from a combination of the two basic scales $\mu_{\mathcal{X}}$ and $\gamma$. So it would appear that at one loop there is a clear way of distinguishing between the masses in the problem. If a lattice computation found a dimension two contribution in channel 1 then that would be a clear indication of an explicit gluon mass term. Though it would not determine whether or not there was also a Gribov mass present too. That could be deduced from the details of other channels and vertices. For the ghost-gluon and quark-gluon vertices we have

$$
\begin{aligned}
& \Sigma_{(1)}^{c c g}\left(p, q, \gamma^{2}, \mu_{\mathcal{X}}^{2}\right)=\Sigma_{(1)}^{c c g}(p, q, 0,0) \\
& +\left[\left[\left[\frac{1}{36} \psi^{\prime}\left(\frac{1}{3}\right)-\frac{11}{32}-\frac{\pi^{2}}{54}+\frac{9}{32} \ln \left[\frac{C_{A} \gamma^{4}}{\mu^{4}}\right]\right] \mu_{\mathcal{X}}^{2}\right.\right. \\
& +\frac{9}{32} \sqrt{\left[\mu_{\mathcal{X}}^{4}-4 C_{A} \gamma^{4}\right]} \ln \left[\frac{\mu_{\mathcal{X}_{+}}^{2}}{\mu_{\mathcal{X}_{-}}^{2}}\right] \\
& \left.+\frac{9 C_{A} \gamma^{4}}{16 \sqrt{\left[\mu_{\mathcal{X}}^{4}-4 C_{A} \gamma^{4}\right]}} \ln \left[\frac{\mu_{\mathcal{X}_{+}}^{2}}{\mu_{\mathcal{X}_{-}}^{2}}\right]\right] \frac{C_{A}}{\mu^{2}} \\
& +\left[\frac{5}{48} \mu_{\mathcal{X}}^{2} \sqrt{\left[\mu_{\mathcal{X}}^{4}-4 C_{A} \gamma^{4}\right]} \ln \left[\frac{\mu_{\mathcal{X}_{+}}^{2}}{\mu_{\mathcal{X}_{-}}^{2}}\right]\right. \\
& +\left[\frac{83}{576}-\frac{\pi^{2}}{54}+\frac{1}{36} \psi^{\prime}\left(\frac{1}{3}\right)+\frac{5}{48} \ln \left[\frac{C_{A} \gamma^{4}}{\mu^{4}}\right]\right] \mu_{\mathcal{X}}^{4} \\
& +\frac{7 C_{A} \mu_{\mathcal{X}}^{2} \gamma^{4}}{24 \sqrt{\left[\mu_{\mathcal{X}}^{4}-4 C_{A} \gamma^{4}\right]}} \ln \left[\frac{\mu_{\mathcal{X}_{+}}^{2}}{\mu_{\mathcal{X}_{-}}^{2}}\right] \\
& \left.+\left[\frac{\pi^{2}}{216}-\frac{377}{2304}-\frac{1}{144} \psi^{\prime}\left(\frac{1}{3}\right)+\frac{1}{12} \ln \left[\frac{C_{A} \gamma^{4}}{\mu^{4}}\right]\right] C_{A} \gamma^{4}\right] \frac{C_{A}}{\mu^{4}}
\end{aligned}
$$




$$
\begin{aligned}
& \left.+O\left(\frac{\mu_{\mathcal{X}}^{6}}{\mu^{6}}\right)\right] a+O\left(a^{2}\right) \\
& \Sigma_{(2)}^{c c g}\left(p, q, \gamma^{2}, \mu_{\mathcal{X}}^{2}\right)=\Sigma_{(2)}^{c c g}(p, q, 0,0) \\
& +\left[\left[\left[\frac{1}{18} \psi^{\prime}\left(\frac{1}{3}\right)+\frac{1}{8}-\frac{\pi^{2}}{27}-\frac{3}{16} \ln \left[\frac{C_{A} \gamma^{4}}{\mu^{4}}\right]\right] \mu_{\mathcal{X}}^{2}\right.\right. \\
& -\frac{3}{16} \sqrt{\left[\mu_{\mathcal{X}}^{4}-4 C_{A} \gamma^{4}\right]} \ln \left[\frac{\mu_{\mathcal{X}_{+}}^{2}}{\mu_{\mathcal{X}_{-}}^{2}}\right] \\
& \left.-\frac{3 C_{A} \gamma^{4}}{8 \sqrt{\left[\mu_{\mathcal{X}}^{4}-4 C_{A} \gamma^{4}\right]}} \ln \left[\frac{\mu_{\mathcal{X}_{+}}^{2}}{\mu_{\mathcal{X}_{-}}^{2}}\right]\right] \frac{C_{A}}{\mu^{2}} \\
& +\left[-\frac{1}{6} \mu_{\mathcal{X}}^{2} \sqrt{\left[\mu_{\mathcal{X}}^{4}-4 C_{A} \gamma^{4}\right]} \ln \left[\frac{\mu_{\mathcal{X}_{+}}^{2}}{\mu_{\mathcal{X}_{-}}^{2}}\right]\right. \\
& +\left[\frac{13}{576}+\frac{\pi^{2}}{54}-\frac{1}{36} \psi^{\prime}\left(\frac{1}{3}\right)-\frac{1}{6} \ln \left[\frac{C_{A} \gamma^{4}}{\mu^{4}}\right]\right] \mu_{\mathcal{X}}^{4} \\
& -\frac{17 C_{A} \mu_{\mathcal{X}}^{2} \gamma^{4}}{48 \sqrt{\left[\mu_{\mathcal{X}}^{4}-4 C_{A} \gamma^{4}\right]}} \ln \left[\frac{\mu_{\mathcal{X}_{+}}^{2}}{\mu_{\mathcal{X}_{-}}^{2}}\right] \\
& \left.+\left[\frac{1}{144} \psi^{\prime}\left(\frac{1}{3}\right)-\frac{\pi^{2}}{216}-\frac{7}{2304}-\frac{1}{48} \ln \left[\frac{C_{A} \gamma^{4}}{\mu^{4}}\right]\right] C_{A} \gamma^{4}\right] \frac{C_{A}}{\mu^{4}} \\
& \left.+O\left(\frac{\mu_{\mathcal{X}}^{6}}{\mu^{6}}\right)\right] a+O\left(a^{2}\right)
\end{aligned}
$$

and

$$
\begin{aligned}
& \Sigma_{(1)}^{q q g}\left(p, q, \gamma^{2}, \mu_{\mathcal{X}}^{2}\right)=\Sigma_{(1)}^{q q g}(p, q, 0,0) \\
& +\left[\left[\left[-\frac{3 C_{A} \gamma^{4}}{4 \sqrt{\left[\mu_{\mathcal{X}}^{4}-4 C_{A} \gamma^{4}\right]}} \ln \left[\frac{\mu_{\mathcal{X}_{+}}^{2}}{\mu_{\mathcal{X}_{-}}^{2}}\right]\right.\right.\right. \\
& +\left[\frac{7}{8}+\frac{10 \pi^{2}}{27}-\frac{5}{9} \psi^{\prime}\left(\frac{1}{3}\right)-\frac{3}{8} \ln \left[\frac{C_{A} \gamma^{4}}{\mu^{4}}\right]\right] \mu_{\mathcal{X}}^{2} \\
& \left.-\frac{3}{8} \sqrt{\left[\mu_{\mathcal{X}}^{4}-4 C_{A} \gamma^{4}\right]} \ln \left[\frac{\mu_{\mathcal{X}_{+}}^{2}}{\mu_{\mathcal{X}_{-}}^{2}}\right]\right] C_{F} \\
& +\left[\left[\frac{5}{16}-\frac{13 \pi^{2}}{27}+\frac{13}{18} \psi^{\prime}\left(\frac{1}{3}\right)-\frac{3}{16} \ln \left[\frac{C_{A} \gamma^{4}}{\mu^{4}}\right]\right] \mu_{\mathcal{X}}^{2}\right. \\
& -\frac{3}{16} \sqrt{\left[\mu_{\mathcal{X}}^{4}-4 C_{A} \gamma^{4}\right]} \ln \left[\frac{\mu_{\mathcal{X}_{+}}^{2}}{\mu_{\mathcal{X}_{-}}^{2}}\right] \\
& \left.\left.-\frac{3 C_{A} \gamma^{4}}{8 \sqrt{\left[\mu_{\mathcal{X}}^{4}-4 C_{A} \gamma^{4}\right]}} \ln \left[\frac{\mu_{\mathcal{X}_{+}}^{2}}{\mu_{\mathcal{X}_{-}}^{2}}\right]\right] C_{A}\right] \frac{1}{\mu^{2}} \\
& +\left[\left[\frac{2 C_{A} \mu_{\mathcal{X}}^{2} \gamma^{4}}{3 \sqrt{\left[\mu_{\mathcal{X}}^{4}-4 C_{A} \gamma^{4}\right]}} \ln \left[\frac{\mu_{\mathcal{X}_{+}}^{2}}{\mu_{\mathcal{X}_{-}}^{2}}\right]\right.\right. \\
& +\left[\frac{67}{36}+\frac{8 \pi^{2}}{27}-\frac{4}{9} \psi^{\prime}\left(\frac{1}{3}\right)-\frac{2}{3} \ln \left[\frac{C_{A} \gamma^{4}}{\mu^{4}}\right]\right] C_{A} \gamma^{4}
\end{aligned}
$$




$$
\begin{aligned}
& +\frac{2}{3} \mu_{\mathcal{X}}^{2} \sqrt{\left[\mu_{\mathcal{X}}^{4}-4 C_{A} \gamma^{4}\right]} \ln \left[\frac{\mu_{\mathcal{X}_{+}}^{2}}{\mu_{\mathcal{X}_{-}}^{2}}\right] \\
& \left.+\left[\frac{4}{9} \psi^{\prime}\left(\frac{1}{3}\right)-\frac{67}{36}-\frac{8 \pi^{2}}{27}+\frac{2}{3} \ln \left[\frac{C_{A} \gamma^{4}}{\mu^{4}}\right]\right] \mu_{\mathcal{X}}^{4}\right] C_{F} \\
& +\left[-\frac{49}{48} \mu_{\mathcal{X}}^{2} \sqrt{\left[\mu_{\mathcal{X}}^{4}-4 C_{A} \gamma^{4}\right]} \ln \left[\frac{\mu_{\mathcal{X}_{+}}^{2}}{\mu_{\mathcal{X}_{-}}^{2}}\right]\right. \\
& +\left[\frac{661}{288}+\frac{8 \pi^{2}}{27}-\frac{4}{9} \psi^{\prime}\left(\frac{1}{3}\right)-\frac{49}{48} \ln \left[\frac{C_{A} \gamma^{4}}{\mu^{4}}\right]\right] \mu_{\mathcal{X}}^{4} \\
& -\frac{67 C_{A} \mu_{\mathcal{X}}^{2} \gamma^{4}}{48 \sqrt{\left[\mu_{\mathcal{X}}^{4}-4 C_{A} \gamma^{4}\right]}} \ln \left[\frac{\mu_{\mathcal{X}_{+}}^{2}}{\mu_{\mathcal{X}_{-}}^{2}}\right] \\
& \left.\left.+\left[\frac{11}{18} \psi^{\prime}\left(\frac{1}{3}\right)-\frac{2941}{1152}-\frac{11 \pi^{2}}{27}+\frac{31}{48} \ln \left[\frac{C_{A} \gamma^{4}}{\mu^{4}}\right]\right] C_{A} \gamma^{4}\right] C_{A}\right] \frac{1}{\mu^{4}} \\
& \left.+O\left(\frac{\mu_{\mathcal{X}}^{6}}{\mu^{6}}\right)\right] a+O\left(a^{2}\right) \\
& \Sigma_{(2)}^{q q g}\left(p, q, \gamma^{2}, \mu_{\mathcal{X}}^{2}\right)=\Sigma_{(5)}^{q q g}\left(p, q, \gamma^{2}, \mu_{\mathcal{X}}^{2}\right) \\
& =\Sigma_{(2)}^{q q g}(p, q, 0,0) \\
& +\left[\left[\left[\left[\frac{5}{3}+\frac{16 \pi^{2}}{27}-\frac{8}{9} \psi^{\prime}\left(\frac{1}{3}\right)\right] \mu_{\mathcal{X}}^{2}\right] C_{F}\right.\right. \\
& +\left[\left[\frac{7}{48}-\frac{16 \pi^{2}}{27}+\frac{8}{9} \psi^{\prime}\left(\frac{1}{3}\right)+\frac{3}{16} \ln \left[\frac{C_{A} \gamma^{4}}{\mu^{4}}\right]\right] \mu_{\mathcal{X}}^{2}\right. \\
& +\frac{3}{16} \sqrt{\left[\mu_{\mathcal{X}}^{4}-4 C_{A} \gamma^{4}\right]} \ln \left[\frac{\mu_{\mathcal{X}_{+}}^{2}}{\mu_{\mathcal{X}_{-}}^{2}}\right] \\
& \left.\left.+\frac{3 C_{A} \gamma^{4}}{8 \sqrt{\left[\mu_{\mathcal{X}}^{4}-4 C_{A} \gamma^{4}\right]}} \ln \left[\frac{\mu_{\mathcal{X}_{+}}^{2}}{\mu_{\mathcal{X}_{-}}^{2}}\right]\right] C_{A}\right] \frac{1}{\mu^{2}} \\
& +\left[\left[\frac{5 C_{A} \mu_{\mathcal{X}}^{2} \gamma^{4}}{6 \sqrt{\left[\mu_{\mathcal{X}}^{4}-4 C_{A} \gamma^{4}\right]}} \ln \left[\frac{\mu_{\mathcal{X}_{+}}^{2}}{\mu_{\mathcal{X}_{-}}^{2}}\right]\right.\right. \\
& +\left[\frac{43}{18}+\frac{16 \pi^{2}}{27}-\frac{8}{9} \psi^{\prime}\left(\frac{1}{3}\right)-\frac{5}{6} \ln \left[\frac{C_{A} \gamma^{4}}{\mu^{4}}\right]\right] C_{A} \gamma^{4} \\
& +\frac{5}{6} \mu_{\mathcal{X}}^{2} \sqrt{\left[\mu_{\mathcal{X}}^{4}-4 C_{A} \gamma^{4}\right]} \ln \left[\frac{\mu_{\mathcal{X}_{+}}^{2}}{\mu_{\mathcal{X}_{-}}^{2}}\right] \\
& \left.+\left[\frac{8}{9} \psi^{\prime}\left(\frac{1}{3}\right)-\frac{43}{18}-\frac{16 \pi^{2}}{27}+\frac{5}{6} \ln \left[\frac{C_{A} \gamma^{4}}{\mu^{4}}\right]\right] \mu_{\mathcal{X}}^{4}\right] C_{F} \\
& +\left[-\frac{79}{48} \mu_{\mathcal{X}}^{2} \sqrt{\left[\mu_{\mathcal{X}}^{4}-4 C_{A} \gamma^{4}\right]} \ln \left[\frac{\mu_{\mathcal{X}_{+}}^{2}}{\mu_{\mathcal{X}_{-}}^{2}}\right]\right. \\
& +\left[\frac{295}{72}+\frac{16 \pi^{2}}{27}-\frac{8}{9} \psi^{\prime}\left(\frac{1}{3}\right)-\frac{79}{48} \ln \left[\frac{C_{A} \gamma^{4}}{\mu^{4}}\right]\right] \mu_{\mathcal{X}}^{4} \\
& -\frac{307 C_{A} \mu_{\mathcal{X}}^{2} \gamma^{4}}{192 \sqrt{\left[\mu_{\mathcal{X}}^{4}-4 C_{A} \gamma^{4}\right]}} \ln \left[\frac{\mu_{\mathcal{X}_{+}}^{2}}{\mu_{\mathcal{X}_{-}}^{2}}\right]
\end{aligned}
$$




$$
\begin{aligned}
& \left.\left.+\left[\frac{14}{9} \psi^{\prime}\left(\frac{1}{3}\right)-\frac{1351}{288}-\frac{28 \pi^{2}}{27}+\frac{325}{192} \ln \left[\frac{C_{A} \gamma^{4}}{\mu^{4}}\right]\right] C_{A} \gamma^{4}\right] C_{A}\right] \frac{1}{\mu^{4}} \\
& \left.+O\left(\frac{\mu_{\mathcal{X}}^{6}}{\mu^{6}}\right)\right] a+O\left(a^{2}\right) \\
& \Sigma_{(3)}^{q q g}\left(p, q, \gamma^{2}, \mu_{\mathcal{X}}^{2}\right)=\Sigma_{(4)}^{q q g}\left(p, q, \gamma^{2}, \mu_{\mathcal{X}}^{2}\right) \\
& =\Sigma_{(3)}^{q q g}(p, q, 0,0) \\
& +\left[\left[\left[\frac{3 C_{A} \gamma^{4}}{2 \sqrt{\left[\mu_{\mathcal{X}}^{4}-4 C_{A} \gamma^{4}\right]}} \ln \left[\frac{\mu_{\mathcal{X}_{+}}^{2}}{\mu_{\mathcal{X}_{-}}^{2}}\right]\right.\right.\right. \\
& +\left[\frac{19}{12}+\frac{4 \pi^{2}}{27}-\frac{2}{9} \psi^{\prime}\left(\frac{1}{3}\right)+\frac{3}{4} \ln \left[\frac{C_{A} \gamma^{4}}{\mu^{4}}\right]\right] \mu_{\mathcal{X}}^{2} \\
& \left.+\frac{3}{4} \sqrt{\left[\mu_{\mathcal{X}}^{4}-4 C_{A} \gamma^{4}\right]} \ln \left[\frac{\mu_{\mathcal{X}_{+}}^{2}}{\mu_{\mathcal{X}_{-}}^{2}}\right]\right] C_{F} \\
& +\left[\left[\frac{29}{48}-\frac{4 \pi^{2}}{27}+\frac{2}{9} \psi^{\prime}\left(\frac{1}{3}\right)-\frac{3}{16} \ln \left[\frac{C_{A} \gamma^{4}}{\mu^{4}}\right]\right] \mu_{\mathcal{X}}^{2}\right. \\
& -\frac{3}{16} \sqrt{\left[\mu_{\mathcal{X}}^{4}-4 C_{A} \gamma^{4}\right]} \ln \left[\frac{\mu_{\mathcal{X}_{+}}^{2}}{\mu_{\mathcal{X}_{-}}^{2}}\right] \\
& \left.\left.-\frac{3 C_{A} \gamma^{4}}{8 \sqrt{\left[\mu_{\mathcal{X}}^{4}-4 C_{A} \gamma^{4}\right]}} \ln \left[\frac{\mu_{\mathcal{X}_{+}}^{2}}{\mu_{\mathcal{X}_{-}}^{2}}\right]\right] C_{A}\right] \frac{1}{\mu^{2}} \\
& +\left[\left[-\frac{C_{A} \mu_{\mathcal{X}}^{2} \gamma^{4}}{2 \sqrt{\left[\mu_{\mathcal{X}}^{4}-4 C_{A} \gamma^{4}\right]}} \ln \left[\frac{\mu_{\mathcal{X}_{+}}^{2}}{\mu_{\mathcal{X}_{-}}^{2}}\right]+\left[\frac{1}{2} \ln \left[\frac{C_{A} \gamma^{4}}{\mu^{4}}\right]-\frac{4}{3}\right] C_{A} \gamma^{4}\right.\right. \\
& \left.-\frac{1}{2} \mu_{\mathcal{X}}^{2} \sqrt{\left[\mu_{\mathcal{X}}^{4}-4 C_{A} \gamma^{4}\right]} \ln \left[\frac{\mu_{\mathcal{X}_{+}}^{2}}{\mu_{\mathcal{X}_{-}}^{2}}\right]+\left[\frac{4}{3}-\frac{1}{2} \ln \left[\frac{C_{A} \gamma^{4}}{\mu^{4}}\right]\right] \mu_{\mathcal{X}}^{4}\right] C_{F} \\
& +\left[-\frac{25}{48} \mu_{\mathcal{X}}^{2} \sqrt{\left[\mu_{\mathcal{X}}^{4}-4 C_{A} \gamma^{4}\right]} \ln \left[\frac{\mu_{\mathcal{X}_{+}}^{2}}{\mu_{\mathcal{X}_{-}}^{2}}\right]\right. \\
& +\left[\frac{65}{144}-\frac{25}{48} \ln \left[\frac{C_{A} \gamma^{4}}{\mu^{4}}\right]\right] \mu_{\mathcal{X}}^{4}-\frac{91 C_{A} \mu_{\mathcal{X}}^{2} \gamma^{4}}{192 \sqrt{\left[\mu_{\mathcal{X}}^{4}-4 C_{A} \gamma^{4}\right]}} \ln \left[\frac{\mu_{\mathcal{X}_{+}}^{2}}{\mu_{\mathcal{X}_{-}}^{2}}\right] \\
& \left.\left.+\left[\frac{2}{3} \psi^{\prime}\left(\frac{1}{3}\right)-\frac{917}{576}-\frac{4 \pi^{2}}{9}+\frac{109}{192} \ln \left[\frac{C_{A} \gamma^{4}}{\mu^{4}}\right]\right] C_{A} \gamma^{4}\right] C_{A}\right] \frac{1}{\mu^{4}} \\
& \left.+O\left(\frac{\mu_{\mathcal{X}}^{6}}{\mu^{6}}\right)\right] a+O\left(a^{2}\right) \\
& \Sigma_{(6)}^{q q g}\left(p, q, \gamma^{2}, \mu_{\mathcal{X}}^{2}\right)=\Sigma_{(6)}^{q q g}(p, q, 0,0) \\
& +\left[\left[\left[-\frac{3 C_{A} \gamma^{4}}{2 \sqrt{\left[\mu_{\mathcal{X}}^{4}-4 C_{A} \gamma^{4}\right]}} \ln \left[\frac{\mu_{\mathcal{X}_{+}}^{2}}{\mu_{\mathcal{X}_{-}}^{2}}\right]\right.\right.\right. \\
& +\left[\frac{7}{4}+\frac{4 \pi^{2}}{9}-\frac{2}{3} \psi^{\prime}\left(\frac{1}{3}\right)-\frac{3}{4} \ln \left[\frac{C_{A} \gamma^{4}}{\mu^{4}}\right]\right] \mu_{\mathcal{X}}^{2} \\
& \left.-\frac{3}{4} \sqrt{\left[\mu_{\mathcal{X}}^{4}-4 C_{A} \gamma^{4}\right]} \ln \left[\frac{\mu_{\mathcal{X}_{+}}^{2}}{\mu_{\mathcal{X}_{-}}^{2}}\right]\right] C_{F}
\end{aligned}
$$




$$
\begin{aligned}
& +\left[\left[\frac{7}{9} \psi^{\prime}\left(\frac{1}{3}\right)-\frac{27}{8}-\frac{14 \pi^{2}}{27}+\frac{9}{8} \ln \left[\frac{C_{A} \gamma^{4}}{\mu^{4}}\right]\right] \mu_{\mathcal{X}}^{2}\right. \\
& +\frac{9}{8} \sqrt{\left[\mu_{\mathcal{X}}^{4}-4 C_{A} \gamma^{4}\right]} \ln \left[\frac{\mu_{\mathcal{X}_{+}}^{2}}{\mu_{\mathcal{X}_{-}}^{2}}\right] \\
& \left.\left.+\frac{9 C_{A} \gamma^{4}}{4 \sqrt{\left[\mu_{\mathcal{X}}^{4}-4 C_{A} \gamma^{4}\right]}} \ln \left[\frac{\mu_{\mathcal{X}_{+}}^{2}}{\mu_{\mathcal{X}_{-}}^{2}}\right]\right] C_{A}\right] \frac{1}{\mu^{2}} \\
& +\left[\left[\frac{C_{A} \mu_{\mathcal{X}}^{2} \gamma^{4}}{3 \sqrt{\left[\mu_{\mathcal{X}}^{4}-4 C_{A} \gamma^{4}\right]}} \ln \left[\frac{\mu_{\mathcal{X}_{+}}^{2}}{\mu_{\mathcal{X}_{-}}^{2}}\right]+\left[\frac{31}{18}-\frac{1}{3} \ln \left[\frac{C_{A} \gamma^{4}}{\mu^{4}}\right]\right] C_{A} \gamma^{4}\right.\right. \\
& \left.+\frac{1}{3} \mu_{\mathcal{X}}^{2} \sqrt{\left[\mu_{\mathcal{X}}^{4}-4 C_{A} \gamma^{4}\right]} \ln \left[\frac{\mu_{\mathcal{X}_{+}}^{2}}{\mu_{\mathcal{X}_{-}}^{2}}\right]+\left[\frac{1}{3} \ln \left[\frac{C_{A} \gamma^{4}}{\mu^{4}}\right]-\frac{31}{18}\right] \mu_{\mathcal{X}}^{4}\right] C_{F} \\
& +\left[\frac{17}{24} \mu_{\mathcal{X}}^{2} \sqrt{\left[\mu_{\mathcal{X}}^{4}-4 C_{A} \gamma^{4}\right]} \ln \left[\frac{\mu_{\mathcal{X}_{+}}^{2}}{\mu_{\mathcal{X}_{-}}^{2}}\right]+\left[\frac{223}{144}+\frac{17}{24} \ln \left[\frac{C_{A} \gamma^{4}}{\mu^{4}}\right]\right] \mu_{\mathcal{X}}^{4}\right. \\
& +\frac{35 C_{A} \mu_{\mathcal{X}}^{2} \gamma^{4}}{24 \sqrt{\left[\mu_{\mathcal{X}}^{4}-4 C_{A} \gamma^{4}\right]}} \ln \left[\frac{\mu_{\mathcal{X}_{+}}^{2}}{\mu_{\mathcal{X}_{-}}^{2}}\right] \\
& \left.\left.\left.+\left[\frac{1}{24} \ln \left[\frac{C_{A} \gamma^{4}}{\mu^{4}}\right]-\frac{991}{576}\right] C_{A} \gamma^{4}\right] C_{A}\right] \frac{1}{\mu^{4}}+O\left(\frac{\mu_{\mathcal{X}}^{6}}{\mu^{6}}\right)\right] a \\
& +O\left(a^{2}\right) \text {. }
\end{aligned}
$$

These are considerably more complicated than their $\mathcal{Q}$ and $\mathcal{R}$ counterparts. However, the key point in this respect both here and in previous results sections is that it is the actual mass scale, no matter how complicated it is in each underlying theory, which is ultimately what the lattice will observe. We note that as a check for each of the three vertices the pure gluon mass expressions are obtained in the $\gamma^{2} \rightarrow 0$ limit in each case.

\section{Discussion.}

We conclude by recalling that we have analysed the one loop corrections to the 3-point vertices of QCD at the symmetric subtraction point in the Landau gauge using the Gribov-Zwanziger Lagrangian and its extensions which incorporate the Gribov problem. The main motivation was to examine the power corrections to the amplitudes in order to see whether the leading correction was dimension two or four. While [1] focused on an effective coupling constant derived from 2 and 3 -point functions and examined the deviations from expected behaviour, we have concentrated on just the vertices themselves. This is because the effective coupling constant definition involved the behaviour of the propagator form factors which have been shown in the Gribov-Zwanziger case to have dimension two corrections, [51. These would therefore dominate in an effective coupling and the leading vertex correction behaviour would not be distinguishable. This is important since we have shown that in certain amplitudes the leading correction is dimension four and not dimension two. Indeed for any asymmetric momentum configuration for the three vertex functions the leading correction is always dimension two. The particular cases where dimension four is leading is in the channel of the triple gluon vertex which corresponds to the Feynman rule of the vertex itself. This is the case not only for the pure Gribov-Zwanziger Lagrangian but also for its extension to either what has been termed the $\mathcal{Q}$ and $\mathcal{R}$ solutions. For a simple gluon mass in the absence of the Gribov mass the triple gluon vertex has a dimension two correction. Since we are at a symmetric subtraction point where the common scale of the 
external legs is not small then examining the deviation from expected perturbative behaviour could provide an important test of the underlying mechanism. For instance, if in measuring the triple gluon vertex channel 1 a power behaviour deviation of dimension two is found then that would rule out a pure Gribov-Zwanziger or $\mathcal{Q}$ and $\mathcal{R}$ explanation. It would not necessarily imply that a pure gluon mass is the underlying reason. This is because there are more complicated extensions of (2.6), [30, not considered here where those propagators could mimic the dimension two correction. We have not introduced these here as we believe of the full set one of $\mathcal{Q}$ or $\mathcal{R}$ is naturally favourable as discussed in [30]. By contrast if a triple gluon vertex measurement indicated that the leading correction was clearly dimension four then that would suggest that the Gribov mass is playing a role. To decipher whether it is the pure Gribov-Zwanziger case would require examination of the strength of the relative corrections and also data on the other vertices. Though this clearly is at a level of fine detail.

It is worth commenting on the current status of lattice measurements of the three vertices at the symmetric point. First, we note that there is only a small amount of data for this point compared with the asymmetric point. For the triple gluon vertex the original indication of a dimension two correction in the effective coupling constant was carried out in [1]. However, in keeping with other analyses of this vertex and the other two it transpires that signals suffer from more noise than the corresponding asymmetric vertex. This is despite the fact that the latter configuration requires a zero momentum limit. Therefore, at this stage one can not yet make any meaningful contact with data on this vertex function in order to see deviations from expected perturbative behaviour aside from the original observations of [1]. Moreover, we note that in [1] the vertex function for the triple gluon vertex was decomposed into only two independent tensors and not three as we have done here. Appendix A provides more details on this point. So even if a comparison with data could be made with our power corrections it is not clear whether this would be meaningful given that the bases are different. Other studies of this vertex include [52, 53]. In the former the four dimensional data, while noisy, show a general decrease towards zero momenta which is reinforced in the latter article. Thus again in these cases a direct comparison with power corrections is not currently viable. For the ghost-gluon vertex the data of [53] does not suffer from as much noise as the triple gluon vertex case. There the main observation is that the ghost-gluon vertex is effectively equivalent to the tree value with a small maximum about $1 \mathrm{GeV}$, [53]. For the quark-gluon vertex there is the additional issue of quenched versus dynamical data. The analysis of [54] was in the quenched approximation and at the symmetric point the data indicate a smoothly rising vertex function. Though the data is noisier than the asymmetric results presented there too and not sufficient in order to perform a comparison with power corrections. Despite this one hope is that with the advances in lattice technology in recent years the focus could return to all of the 3-point functions now that there seems to be a consensus on the zero momentum behaviour of the gluon and ghost 2-point functions.

Finally, we should qualify our overall remarks by noting that we have performed the analysis at one loop. One could regard this as a next to high energy expansion. However, there is no a priori reason why the leading dimension four correction of channel 1 of the triple gluon vertex should persist beyond one loop. The leading two loop correction to this channel could be dimension two. So a more careful test could be that if the leading correction is dimension two but relatively weak compared to the other leading one loop dimension two corrections in other channels then that could be evidence for a non-pure gluon mass explanation. To carry out a two loop extension to the expansion is in principle possible but is beyond the scope of the present paper. The technical calculational tools are clearly available. Though one would have a substantial number of REDUZE databases to build for the two main topologies that occur at two loops, [38], to cover all the non-zero mass distribution possibilities. While a one loop 
observation is by no means a proof it is intriguing that of the three 3-point vertices of QCD it is actually the fully symmetric one in terms of fields when examined in this power expansion specifically at the symmetric subtraction point which should have dimension four as the leading correction.

Acknowledgement. The author thanks Dr. P.A. Boyle for useful discussions.

\section{A Tensor basis.}

In order to assist with the interpretation of the results in this appendix we record the explicit forms of the tensors in the bases for each of the vertices. For the triple gluon vertex the tensors of the original of [38] are

$$
\begin{aligned}
\mathcal{P}_{(1) \mu \nu \sigma}^{\mathrm{ggg}}(p, q) & =\eta_{\mu \nu} p_{\sigma}, \quad \mathcal{P}_{(2) \mu \nu \sigma}^{\mathrm{ggg}}(p, q)=\eta_{\nu \sigma} p_{\mu}, \quad \mathcal{P}_{(3) \mu \nu \sigma}^{\mathrm{ggg}}(p, q)=\eta_{\sigma \mu} p_{\nu} \\
\mathcal{P}_{(4) \mu \nu \sigma}^{\mathrm{ggg}}(p, q) & =\eta_{\mu \nu} q_{\sigma}, \quad \mathcal{P}_{(5) \mu \nu \sigma}^{\mathrm{ggg}}(p, q)=\eta_{\nu \sigma} q_{\mu}, \quad \mathcal{P}_{(6) \mu \nu \sigma}^{\mathrm{ggg}}(p, q)=\eta_{\sigma \mu} q_{\nu} \\
\mathcal{P}_{(7) \mu \nu \sigma}^{\mathrm{ggg}}(p, q) & =\frac{1}{\mu^{2}} p_{\mu} p_{\nu} p_{\sigma}, \quad \mathcal{P}_{(8) \mu \nu \sigma}^{\mathrm{ggg}}(p, q)=\frac{1}{\mu^{2}} p_{\mu} p_{\nu} q_{\sigma}, \quad \mathcal{P}_{(9) \mu \nu \sigma}^{\mathrm{ggg}}(p, q)=\frac{1}{\mu^{2}} p_{\mu} q_{\nu} p_{\sigma} \\
\mathcal{P}_{(10) \mu \nu \sigma}^{\mathrm{ggg}}(p, q) & =\frac{1}{\mu^{2}} q_{\mu} p_{\nu} p_{\sigma}, \quad \mathcal{P}_{(11) \mu \nu \sigma}^{\mathrm{ggg}}(p, q)=\frac{1}{\mu^{2}} p_{\mu} q_{\nu} q_{\sigma}, \quad \mathcal{P}_{(12) \mu \nu \sigma}^{\mathrm{ggg}}(p, q)=\frac{1}{\mu^{2}} q_{\mu} p_{\nu} q_{\sigma} \\
\mathcal{P}_{(13) \mu \nu \sigma}^{\mathrm{ggg}}(p, q) & =\frac{1}{\mu^{2}} q_{\mu} q_{\nu} p_{\sigma}, \quad \mathcal{P}_{(14) \mu \nu \sigma}^{\mathrm{ggg}}(p, q)=\frac{1}{\mu^{2}} q_{\mu} q_{\nu} q_{\sigma}
\end{aligned}
$$

where the first six correspond to the terms of the original vertex in the Lagrangian. However, from explicit calculations, [38, it transpires that the basis can be compactified into three independent combinations which we define to be

$$
\begin{aligned}
& \tilde{\mathcal{P}}_{(1) \mu \nu \sigma}^{g g g}(p, q)=\eta_{\mu \nu} p_{\sigma}-\eta_{\mu \nu} q_{\sigma}-2 \eta_{\mu \sigma} p_{\nu}-\eta_{\sigma \mu} q_{\nu}+\eta_{\nu \sigma} p_{\mu}+2 \eta_{\nu \sigma} q_{\mu} \\
& \tilde{\mathcal{P}}_{(2) \mu \nu \sigma}^{g g g}(p, q)=\left[2 p_{\mu} p_{\nu} p_{\sigma}+p_{\mu} q_{\nu} p_{\sigma}-p_{\mu} q_{\nu} q_{\sigma}+2 q_{\mu} p_{\nu} p_{\sigma}-2 q_{\mu} p_{\nu} q_{\sigma}-2 q_{\mu} q_{\nu} q_{\sigma}\right] \frac{1}{2 \mu^{2}} \\
& \tilde{\mathcal{P}}_{(3) \mu \nu \sigma}^{g g g}(p, q)=\left[p_{\mu} p_{\nu} q_{\sigma}-q_{\mu} p_{\nu} p_{\sigma}+q_{\mu} p_{\nu} q_{\sigma}-q_{\mu} q_{\nu} p_{\sigma}\right] \frac{1}{\mu^{2}} .
\end{aligned}
$$

The first again is the triple gluon vertex when one sets $r=-p-q$. In the analysis of [1] only two tensors are defined. One corresponds to the first and the second is a linear combination of the other two. More specifically the second tensor of [1] is proportional to

$$
\tilde{\mathcal{P}}_{(2) \mu \nu \sigma}^{g g g}(p, q)-\tilde{\mathcal{P}}_{(3) \mu \nu \sigma}^{g g g}(p, q)=-\frac{1}{2 \mu^{2}}(q-r)_{\mu}(r-p)_{\nu}(p-q)_{\sigma} .
$$

Though the explicit two loop calculations of [38] indicate that this choice of basis is too limited. In order to project out the amplitudes the projection matrix for (A.2) is

$$
\tilde{\mathcal{M}}_{p q}^{\text {ggg }}=-\frac{1}{27(d-2)}\left(\begin{array}{ccc}
3 & 0 & -6 \\
0 & 16(d-2) & 8(d-2) \\
-6 & 8(d-2) & 4(4 d-5)
\end{array}\right)
$$

where the subscript denotes the symmetric point. The corresponding $14 \times 14$ matrix for $\mathcal{P}_{(i) \mu \nu \sigma}^{\text {ggg }}(p, q)$ as well as those for the quark and ghost vertices used here were given in [38] but, for completeness here, the tensors of the latter two respective bases are

$$
\mathcal{P}_{(1) \sigma}^{\mathrm{ccg}}(p, q)=p_{\sigma} \quad, \quad \mathcal{P}_{(2) \sigma}^{\mathrm{ccg}}(p, q)=q_{\sigma}
$$


and

$$
\begin{aligned}
& \mathcal{P}_{(1) \sigma}^{\mathrm{qqg}}(p, q)=\gamma_{\sigma} \quad, \quad \mathcal{P}_{(2) \sigma}^{\mathrm{qqg}}(p, q)=\frac{p_{\sigma} \not p}{\mu^{2}}, \quad \mathcal{P}_{(3) \sigma}^{\mathrm{qqg}}(p, q)=\frac{p_{\sigma} \not h}{\mu^{2}}, \\
& \mathcal{P}_{(4) \sigma}^{\mathrm{qqg}}(p, q)=\frac{q_{\sigma} \not p}{\mu^{2}} \quad, \quad \mathcal{P}_{(5) \sigma}^{\mathrm{qqg}}(p, q)=\frac{q_{\sigma} \not}{\mu^{2}}, \quad \mathcal{P}_{(6) \sigma}^{\mathrm{qqg}}(p, q)=\frac{1}{\mu^{2}} \Gamma_{(3) \sigma p q} .
\end{aligned}
$$

We use the convention that when an external momentum is contracted with a Lorentz index then the dummy index is replaced by that momentum.

\section{B Leading order amplitudes.}

In this appendix for completeness we record the explicit values for the various one loop amplitudes of each vertex at the symmetric point in the absence of the Gribov mass. They were computed originally in [55]. We have

$$
\begin{aligned}
\tilde{\Sigma}_{(1)}^{g g g}(p, q, 0,0)= & -1+\left[\left[\frac{16}{27} \psi^{\prime}\left(\frac{1}{3}\right)-2-\frac{32}{81} \pi^{2}\right] T_{F} N_{f}+\left[\frac{3}{8}+\frac{23}{162} \pi^{2}-\frac{23}{108} \psi^{\prime}\left(\frac{1}{3}\right)\right] C_{A}\right] a \\
& +O\left(a^{2}\right) \\
\tilde{\Sigma}_{(2)}^{g g g}(p, q, 0,0)= & {\left[\left[\frac{16}{27}+\frac{128}{243} \pi^{2}-\frac{64}{81} \psi^{\prime}\left(\frac{1}{3}\right)\right] T_{F} N_{f}+\left[\frac{97}{108}-\frac{67}{243} \pi^{2}+\frac{67}{162} \psi^{\prime}\left(\frac{1}{3}\right)\right] C_{A}\right] a } \\
& +O\left(a^{2}\right) \\
\tilde{\Sigma}_{(3)}^{g g g}(p, q, 0,0)= & {\left[\left[\frac{64}{243} \pi^{2}-\frac{28}{27}-\frac{32}{81} \psi^{\prime}\left(\frac{1}{3}\right)\right] T_{F} N_{f}+\left[\frac{67}{54}-\frac{56}{243} \pi^{2}+\frac{28}{81} \psi^{\prime}\left(\frac{1}{3}\right)\right] C_{A}\right] a } \\
& +O\left(a^{2}\right)
\end{aligned}
$$

for the triple gluon vertex. We note that we have checked that these expressions agree with those

derived in [38] after converting the amplitudes of [38] to the basis used here. The amplitudes for the ghost-gluon vertex are

$$
\begin{aligned}
& \Sigma_{(1)}^{c c g}(p, q, 0,0)=-1+\left[\frac{5}{108} \pi^{2}-\frac{5}{72} \psi^{\prime}\left(\frac{1}{3}\right)-\frac{1}{2}\right] C_{A} a+O\left(a^{2}\right) \\
& \Sigma_{(2)}^{c c g}(p, q, 0,0)=\left[\frac{5}{72} \psi^{\prime}\left(\frac{1}{3}\right)-\frac{5}{108} \pi^{2}+\frac{1}{4}\right] C_{A} a+O\left(a^{2}\right)
\end{aligned}
$$

and those for the quark-gluon vertex are

$$
\begin{aligned}
\Sigma_{(1)}^{q q g}(p, q, 0,0)= & 1+\left[\left[\frac{13}{4}+\frac{13}{54} \pi^{2}-\frac{13}{36} \psi^{\prime}\left(\frac{1}{3}\right)\right] C_{A}+\left[\frac{2}{9} \psi^{\prime}\left(\frac{1}{3}\right)-\frac{4}{27} \pi^{2}-2\right] C_{F}\right] a \\
& +O\left(a^{2}\right) \\
\Sigma_{(2)}^{q q g}(p, q, 0,0)= & \Sigma_{(5)}^{q q g}(p, q, 0,0) \\
= & {\left[\left[\frac{7}{3}+\frac{5}{27} \pi^{2}-\frac{5}{16} \psi^{\prime}\left(\frac{1}{3}\right)\right] C_{A}+\left[\frac{4}{9} \psi^{\prime}\left(\frac{1}{3}\right)-\frac{8}{27} \pi^{2}-\frac{8}{3}\right] C_{F}\right] a+O\left(a^{2}\right) } \\
\Sigma_{(3)}^{q q g}(p, q, 0,0)= & \Sigma_{(4)}^{q q g}(p, q, 0,0) \\
= & {\left[\left[\frac{5}{3}+\frac{2}{27} \pi^{2}-\frac{1}{9} \psi^{\prime}\left(\frac{1}{3}\right)\right] C_{A}-\frac{4}{3} C_{F}\right] a+O\left(a^{2}\right) } \\
\Sigma_{(6)}^{q q g}(p, q, 0,0)= & {\left[\left[\frac{11}{27} \pi^{2}-\frac{11}{18} \psi^{\prime}\left(\frac{1}{3}\right)\right] C_{A}+\left[\frac{4}{9} \psi^{\prime}\left(\frac{1}{3}\right)-\frac{8}{27} \pi^{2}\right] C_{F}\right] a+O\left(a^{2}\right) . }
\end{aligned}
$$

The numerical evaluation of these as well as all the two loop corrections were given in [38]. 


\section{Expansions for various master integrals.}

In this section we present the expansions for several master integrals in powers of $1 / \mu^{2}$. These were established by the methods of [31] which were discussed in section 3. First, we have for one mass scale

$$
\begin{aligned}
I\left(i \sqrt{C_{A}} \gamma^{2}, 0,0\right)= & {\left[\frac{4 \pi^{2}}{9}-\frac{2}{3} \psi^{\prime}\left(\frac{1}{3}\right)\right] \frac{1}{\mu^{2}}-\left[\frac{\pi}{2}+i-\frac{i}{2} \ln \left[\frac{C_{A} \gamma^{4}}{\mu^{4}}\right]\right] \frac{\sqrt{C_{A}} \gamma^{2}}{\mu^{4}} } \\
& -\left[\frac{3}{4}+\frac{1}{4} \ln \left[\frac{C_{A} \gamma^{4}}{\mu^{4}}\right]+\frac{\pi i}{4}\right] \frac{C_{A} \gamma^{4}}{\mu^{6}}+O\left(\frac{\gamma^{6}}{\mu^{8}}\right) .
\end{aligned}
$$

For two non-zero masses we have

$$
\begin{aligned}
I\left(i \sqrt{C_{A}} \gamma^{2}, i \sqrt{C_{A}} \gamma^{2}, 0\right)= & {\left[\frac{4 \pi^{2}}{9}-\frac{2}{3} \psi^{\prime}\left(\frac{1}{3}\right)\right] \frac{1}{\mu^{2}}-\left[\pi+2 i-i \ln \left[\frac{C_{A} \gamma^{4}}{\mu^{4}}\right]\right] \frac{\sqrt{C_{A}} \gamma^{2}}{\mu^{4}} } \\
& -\left[\frac{1}{2}+\frac{3}{2} \ln \left[\frac{C_{A} \gamma^{4}}{\mu^{4}}\right]+\frac{3 \pi i}{2}\right] \frac{C_{A} \gamma^{4}}{\mu^{6}}+O\left(\frac{\gamma^{6}}{\mu^{8}}\right)
\end{aligned}
$$

and

$$
\begin{aligned}
I\left(i \sqrt{C_{A}} \gamma^{2},-i \sqrt{C_{A}} \gamma^{2}, 0\right)= & {\left[\frac{4 \pi^{2}}{9}-\frac{2}{3} \psi^{\prime}\left(\frac{1}{3}\right)\right] \frac{1}{\mu^{2}}-\pi \frac{\sqrt{C_{A}} \gamma^{2}}{\mu^{4}} } \\
& +\left[\frac{1}{2} \ln \left[\frac{C_{A} \gamma^{4}}{\mu^{4}}\right]-\frac{5}{2}\right] \frac{C_{A} \gamma^{4}}{\mu^{6}}+O\left(\frac{\gamma^{6}}{\mu^{8}}\right)
\end{aligned}
$$

which is real as expected. In the pure Gribov-Zwanziger case the main cases with three non-zero entries are

$$
\begin{aligned}
I\left(i \sqrt{C_{A}} \gamma^{2}, i \sqrt{C_{A}} \gamma^{2}, i \sqrt{C_{A}} \gamma^{2}\right)= & {\left[\frac{4 \pi^{2}}{9}-\frac{2}{3} \psi^{\prime}\left(\frac{1}{3}\right)\right] \frac{1}{\mu^{2}} } \\
& -\left[\frac{3 \pi}{2}+3 i-\frac{3 i}{2} \ln \left[\frac{C_{A} \gamma^{4}}{\mu^{4}}\right]\right] \frac{\sqrt{C_{A}} \gamma^{2}}{\mu^{4}} \\
& +\left[\frac{3}{4}-\frac{15}{4} \ln \left[\frac{C_{A} \gamma^{4}}{\mu^{4}}\right]-\frac{15 \pi i}{4}\right] \frac{C_{A} \gamma^{4}}{\mu^{6}}+O\left(\frac{\gamma^{6}}{\mu^{8}}\right)
\end{aligned}
$$

and

$$
\begin{aligned}
I\left(i \sqrt{C_{A}} \gamma^{2}, i \sqrt{C_{A}} \gamma^{2},-i \sqrt{C_{A}} \gamma^{2}\right)= & {\left[\frac{4 \pi^{2}}{9}-\frac{2}{3} \psi^{\prime}\left(\frac{1}{3}\right)\right] \frac{1}{\mu^{2}} } \\
& -\left[\frac{3 \pi}{2}+i-\frac{i}{2} \ln \left[\frac{C_{A} \gamma^{4}}{\mu^{4}}\right]\right] \frac{\sqrt{C_{A}} \gamma^{2}}{\mu^{4}} \\
& +\left[\frac{1}{4} \ln \left[\frac{C_{A} \gamma^{4}}{\mu^{4}}\right]-\frac{13}{4}-\frac{5 \pi i}{4}\right] \frac{C_{A} \gamma^{4}}{\mu^{6}}+O\left(\frac{\gamma^{6}}{\mu^{8}}\right) .
\end{aligned}
$$

For either the $\mathcal{Q}$ or $\mathcal{R}$ solutions we have

$$
\begin{aligned}
I\left(\mu_{\mathcal{I}_{+}}^{2}, \mu_{\mathcal{I}_{-}}^{2}, \mu_{\mathcal{I}}^{2}\right)= & {\left[\frac{4 \pi^{2}}{9}-\frac{2}{3} \psi^{\prime}\left(\frac{1}{3}\right)\right] \frac{1}{\mu^{2}} } \\
& +\left[\mu_{\mathcal{I}_{+}}^{2}\left[\ln \left[\frac{\mu_{\mathcal{I}_{+}}^{2}}{\mu^{2}}\right]-1\right]+\mu_{\mathcal{I}_{-}}^{2}\left[\ln \left[\frac{\mu_{\mathcal{I}_{-}}^{2}}{\mu^{2}}\right]-1\right]+\mu_{\mathcal{I}}^{2}\left[\ln \left[\frac{\mu_{\mathcal{I}}^{2}}{\mu^{2}}\right]-1\right]\right] \frac{1}{\mu^{4}}
\end{aligned}
$$




$$
\begin{aligned}
& +\left[\mu_{\mathcal{I}_{+}}^{4}\left[\frac{1}{2} \ln \left[\frac{\mu_{\mathcal{I}_{+}}^{2}}{\mu^{2}}\right]+\frac{3}{4}\right]+\mu_{\mathcal{I}_{-}}^{4}\left[\frac{1}{2} \ln \left[\frac{\mu_{\mathcal{I}_{-}}^{2}}{\mu^{2}}\right]+\frac{3}{4}\right]\right. \\
& +\mu_{\mathcal{I}}^{4}\left[\frac{1}{2} \ln \left[\frac{\mu_{\mathcal{I}}^{2}}{\mu^{2}}\right]+\frac{3}{4}\right]+\mu_{\mathcal{I}_{+}}^{2} \mu_{\mathcal{I}_{-}}^{2}\left[\ln \left[\frac{\mu_{\mathcal{I}_{+}}^{2}}{\mu^{2}}\right]+\ln \left[\frac{\mu_{\mathcal{I}_{-}}^{2}}{\mu^{2}}\right]-1\right] \\
& +\mu_{\mathcal{I}^{2}}^{2} \mu_{\mathcal{I}_{+}}^{2}\left[\ln \left[\frac{\mu_{\mathcal{I}}^{2}}{\mu^{2}}\right]+\ln \left[\frac{\mu_{\mathcal{I}_{+}}^{2}}{\mu^{2}}\right]-1\right] \\
& \left.\quad+\mu_{\mathcal{I}^{\prime}}^{2} \mu_{\mathcal{I}_{-}}^{2}\left[\ln \left[\frac{\mu_{\mathcal{I}}^{2}}{\mu^{2}}\right]+\ln \left[\frac{\mu_{\mathcal{I}_{-}}^{2}}{\mu^{2}}\right]-1\right]\right] \frac{1}{\mu^{6}}+O\left(\frac{\gamma^{6}}{\mu^{8}}\right)
\end{aligned}
$$

for the case of three distinct non-zero masses. In each case the order symbols are intended to reflect the power of $\mu$ and the numerator factor therein is merely to have the correct overall dimensionful dependence. We have also given the expansion out to powers beyond that which we have indicated we are interested in for the overall vertex functions. This is because in the rearrangement of the numerator scalar products in the original integrals one can be left with terms such as $p^{2}$ and $p q$ which are proportional to $\mu^{2}$. Hence, terms beyond the dimension four ones we are interested in for the overall vertex function need to be retained in the above examples and the expansion of the other basic master integrals.

\section{References.}

[1] Ph. Boucaud, J.P. Leroy, J. Micheli, O. Pène \& C. Roiesnel, JHEP 9810 (1998), 017.

[2] G. Burgio, F. Di Renzo, C. Parrinello \& C. Pittori, hep-ph/9808258.

[3] P. Boucaud, G. Burgio, F. Di Renzo, J.P. Leroy, J. Micheli, C. Parrinello, O. Pène, C. Pittori, J. Rodríguez-Quintero, C. Roiesnel \& K. Sharkey, JHEP 0004 (2000), 006.

[4] F. De Soto \& J. Rodríguez-Quintero, Phys. Rev. D64 (2001), 114003.

[5] Ph. Boucaud, J.P. Leroy, H. Moutarde, J. Micheli, O. Pène, J. Rodríguez-Quintero \& C. Roisenel, JHEP 0201 (2002), 046.

[6] V.I. Zakharov, Nucl. Phys. Proc. Suppl. 164 (2007), 240.

[7] J.M. Cornwall, Phys. Rev. D26 (1982), 1453.

[8] A. Cucchieri \& T. Mendes, PoS LAT2007 (2007), 297.

[9] I.L. Bogolubsky, E.M. Ilgenfritz, M. Müller-Preussker \& A. Sternbeck, PoS LAT2007 (2007), 290.

[10] A. Maas, Phys. Rev. D75 (2007), 116004.

[11] A. Sternbeck, L. von Smekal, D.B. Leinweber \& A.G. Williams, PoS LAT2007 (2007), 304.

[12] I.L. Bogolubsky, E.M. Ilgenfritz, M. Müller-Preussker \& A. Sternbeck, Phys. Lett. B676 (2009), 69.

[13] A. Cucchieri \& T. Mendes, Phys. Rev. Lett. 100 (2008), 241601.

[14] A. Cucchieri \& T. Mendes, Phys. Rev. D78 (2008), 094503.

[15] O. Oliveira \& P.J. Silva, Phys. Rev. D79 (2009), 031501. 
[16] Ph. Boucaud, J.P. Leroy, A.L. Yaounac, J. Micheli, O. Pène \& J. Rodríguez-Quintero, JHEP 0806 (2008), 099.

[17] V.N. Gribov, Nucl. Phys. B139 (1978), 1.

[18] J.C. Taylor, Nucl. Phys. B33 (1971), 436.

[19] D. Zwanziger, Nucl. Phys. B209 (1982), 336.

[20] D. Zwanziger, Nucl. Phys. B321 (1989), 591.

[21] D. Zwanziger, Nucl. Phys. B323 (1989), 513.

[22] G. Dell'Antonio \& D. Zwanziger, Nucl. Phys. B326 (1989), 333.

[23] G. Dell'Antonio \& D. Zwanziger, Commun. Math. Phys. 138 (1991), 291.

[24] D. Zwanziger, Nucl. Phys. B364 (1991), 127.

[25] D. Zwanziger, Nucl. Phys. B378 (1992), 525.

[26] D. Zwanziger, Nucl. Phys. B399 (1993), 477.

[27] D. Zwanziger, Nucl. Phys. B412 (1994), 657.

[28] D. Dudal, S.P. Sorella, N. Vandersickel \& H. Verschelde, Phys. Rev. D77 (2008), 071501.

[29] D. Dudal, J.A. Gracey, S.P. Sorella, N. Vandersickel \& H. Verschelde, Phys. Rev. D78 (2008), 065047.

[30] J.A. Gracey, Phys. Rev. D82 (2010), 085032.

[31] A.I. Davydychev, V.A. Smirnov \& J.B. Tausk, Nucl. Phys. B410 (1993) 325.

[32] J.M. Cornwall, Nucl. Phys. B157 (1979), 392.

[33] D. Zwanziger, Nucl. Phys. B345 (1990), 461.

[34] N. Maggiore \& M. Schaden, Phys. Rev. D50 (1994), 6616.

[35] D. Dudal, R.F. Sobreiro, S.P. Sorella \& H. Verschelde, Phys. Rev. D72 (2005), 014016.

[36] J.A. Gracey, Phys. Lett. B632 (2006), 282; Phys. Lett. B686 (2010), 319.

[37] T. van Ritbergen, A.N. Schellekens \& J.A.M. Vermaseren, Int. J. Mod. Phys. A14 (1999), 41.

[38] J.A. Gracey, Phys. Rev. D84 (2011), 085011.

[39] A.D. Kennedy, J. Math. Phys. 22 (1981), 1330.

[40] A. Bondi, G. Curci, G. Paffuti \& P. Rossi, Ann. Phys. 199 (1990), 268.

[41] A.N. Vasil'ev, S.É. Derkachov \& N.A. Kivel, Theor. Math. Phys. 103 (1995), 487.

[42] A.N. Vasil'ev, M.I. Vyazovskii, S.É. Derkachov \& N.A. Kivel, Theor. Math. Phys. 107 (1996), 441.

[43] A.N. Vasil'ev, M.I. Vyazovskii, S.É. Derkachov \& N.A. Kivel, Theor. Math. Phys. 107 (1996), 710 . 
[44] S. Laporta, Int. J. Mod. Phys. A15 (2000), 5087.

[45] C. Studerus, Comput. Phys. Commun. 181 (2010), 1293.

[46] C.W. Bauer, A. Frink \& R. Kreckel, cs/0004015.

[47] J.A.M. Vermaseren, math-ph/0010025.

[48] P. Nogueira, J. Comput. Phys. 105 (1993), 279.

[49] A.I. Davydychev, J. Phys. A25 (1992), 5587.

[50] G. Curci \& R. Ferrari, Nuovo Cim. A32 (1976), 151.

[51] J.A. Gracey, JHEP 0605 (2006), 052; JHEP 1002 (2010), 078.

[52] A. Cucchieri, A. Maas \& T. Mendes, Phys. Rev. D74 (2006), 014503.

[53] A. Cucchieri, A. Maas \& T. Mendes, Phys. Rev. D77 (2008), 094510.

[54] A. Kızılersü, D.B. Leinweber, J.-I. Skullerud \& A.G. Williams, Eur. Phys. J. C50 (2007), 87.

[55] W. Celmaster \& R.J. Gonsalves, Phys. Rev. D20 (1979), 1420. 